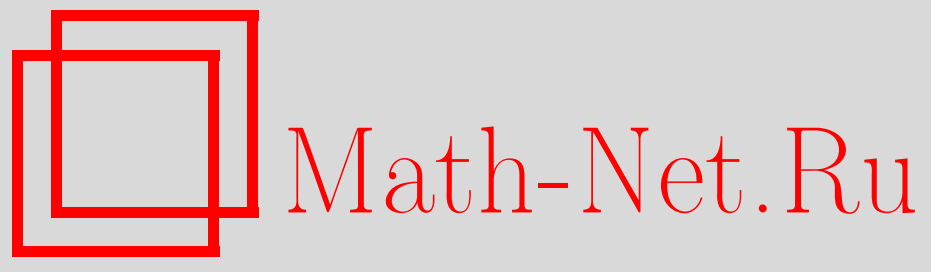

С. М. Гусейн-Заде, Интегрирование по отношению к эйлеровой характеристике и его приложения, УМН, 2010, том 65, выпуск 3, 5-42

DOI: https://doi.org/10.4213/rm9357

Использование Общероссийского математического портала Math-Net.Ru подразумевает, что вы прочитали и согласны с пользовательским соглашением http://www . mathnet.ru/rus/agreement

Параметры загрузки:

IP: 54.162 .127 .20

26 апреля 2023 г., 12:49:53

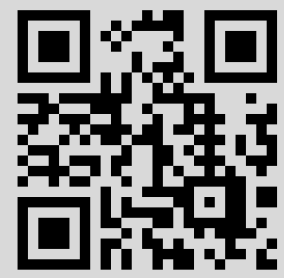




\section{Интегрирование по отношению к эйлеровой характеристике и его приложения}

\section{С. М. Гусейн-Заде}

Обсуждается понятие интегрирования по эйлеровой характеристике и его обобщения: интегрирование по бесконечномерным пространствам дуг или функций, мотивное интегрирование. Описываются приложения этих понятий к вычислению дзета-функций монодромий, рядов Пуанкаре мультииндексных фильтраций, производящих рядов классов некоторых классифицирующих пространств и т. д.

Библиография: 70 названий.

Ключевые слова: эйлерова характеристика, мотивное интегрирование.

\section{СОДЕРЖАНИЕ}

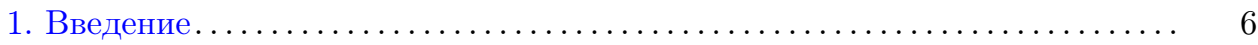

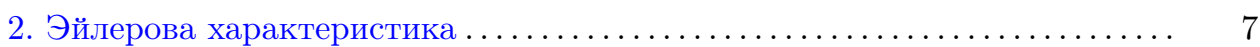

3. Интеграл по отношению к эйлеровой характеристике $\ldots \ldots \ldots \ldots \ldots \ldots$.

4. Некоторые примеры . . . . . . . . . . . . . . . . . . . . . . . 11

5. Ряды Пуанкаре фильтраций и интегралы по эйлеровой характеристике 16

6. Вычисление рядов Пуанкаре через интегралы по эйлеровой характери-

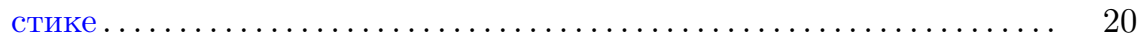

7. Универсальная эйлерова характеристика и мотивная мера на простран-

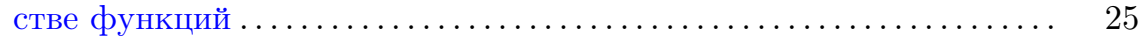

8. Мотивное интегрирование......................... 28

9. Степенная структура над кольцом Гротендика квазипроективных мно-

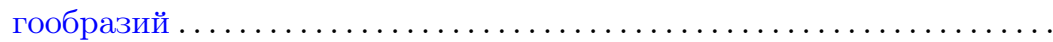

10. Степенная структура над кольцом $K_{0}\left(\mathscr{V}_{\mathbb{C}}\right)$ и производящие ряды классов некоторых конфигурационных пространств .............. 35

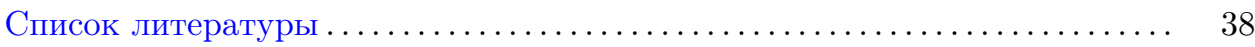

Работа выполнена при поддержке РФФИ (грант № 07-01-00593) и программы "Ведущие научные школы” (грант НШ-709.2008.1). Больша́я часть статьи была написана, когда автор пользовался гостеприимством Международного центра теоретической физики (ICTP), Триест, Италия. 


\section{1. Введение}

Определение интеграла требует наличия меры. Обычно мера - это неотрицательная аддитивная функция на некоторой алгебре множеств. Аддитивность меры $\mu$ означает, что

$$
\mu(X \cup Y)=\mu(X)+\mu(Y)-\mu(X \cap Y)
$$

для множеств $X$ и $Y$ из рассматриваемой алгебры (и, значит, $\mu(X \cup Y)=$ $\mu(X)+\mu(Y)$ для непересекающихся множеств $X$ и $Y)$. Для определения интеграла аддитивность меры совершенно необходима, в то время как требование неотрицательности может быть снято.

Примером аддитивной функции на алгебре множеств является эйлерова характеристика (подходящим образом определенная). Поэтому она может рассматриваться как (неположительная) мера для определения соответствующего понятия интеграла: интеграла по отношению к эйлеровой характеристике. В качестве алгебры множеств, для которых определена эйлерова характеристика, обычно используется алгебра конструктивных подмножеств проективного пространства. [Конструктивное подмножество проективного пространства это конечное объединение квазипроективных (полуалгебраических) множеств. Квазипроективное множество - это разность двух проективных.]

То, что некоторые выражения, содержащие эйлеровы характеристики, могут рассматриваться как своеобразные интегралы, было замечено довольно давно. Однако точное определение и соответствующее название впервые были даны О.Я. Виро в [1]. Там же приведены некоторые приложения этого понятия. Понятие интеграла по отношению к эйлеровой характеристике позволяет как записывать в другой (более компактной) форме известные утверждения, так и формулировать и доказывать новые.

Например, формула Римана-Гурвица для рода разветвленного накрытия комплексной кривой может быть представлена как формула, выражающая этот род в терминах некоторого интеграла по отношению к эйлеровой характеристике по исходной кривой. Формула А'Кампо для дзета-функции классической монодромии ростка функции в терминах разрешения особенности может быть записана в виде интеграла по отношению к эйлеровой характеристике по исключительному дивизору разрешения. В работе будут приведены и другие примеры.

Далеко идущим обобщением понятия интеграла по отношению к эйлеровой характеристике является мотивное интегрирование, введенное М. Концевичем [2] и развитое в работах Я. Денефа и Ф. Лозера [3]-[5], В. Батырева [6]-[9] и др. Можно сказать, что мотивное интегрирование является обобщением интегрирования по отношению к эйлеровой характеристике в двух направлениях. Во-первых, вместо интегрирования по конечномерному (например, квазипроективному) пространству интегрирование ведется по бесконечномерному пространству дуг на многообразии. Во-вторых, в качестве меры используется не обычная эйлерова характеристика (со значениями в кольце $\mathbb{Z}$ целых чисел), а обобщенная (универсальная) эйлерова характеристика со значениями 
в кольце Гротендика квазипроективных многообразий (или, точнее, в его модификации: локализации, пополнении). Мотивное интегрирование позволило доказать несколько трудных утверждений алгебраической геометрии, а также построить ряд новых инвариантов.

Наконец, интегрировать по отношению к эйлеровой характеристике (как обычной, так и обобщенной) можно не только по пространству дуг, но и по некоторым другим бесконечномерным пространствам, на которых может быть определен подходящий аналог меры. Примерами таковых являются пространство ростков функций на особенности аналитического пространства или его проективизация, пространство непараметризованных дуг на многообразии и др. Так, интеграл по отношению к эйлеровой характеристике по проективизации кольца ростков функций оказался эффективным инструментом для вычисления рядов Пуанкаре некоторых естественных фильтраций (одноиндексных и мультииндексных) на этом кольце.

\section{2. Эйлерова характеристика}

Чуть отложим обсуждение вопроса о том, на какой алгебре множеств (или на каких алгебрах множеств) мы будем рассматривать эйлерову характеристику, и обсудим сначала ее аддитивность. В соответствии с одним из обычных определений, эйлерова характеристика пространства $X$ - это альтернированная сумма чисел Бетти этого пространства:

$$
\chi(X)=\sum_{q \geqslant 0}(-1)^{q} \operatorname{dim} H^{q}(X) .
$$

Здесь группы когомологий $H^{q}(X)$ пространства $X$ рассматриваются с коэффициентами в некотором поле, например, в поле вещественных чисел $\mathbb{R}$. Однако, если понимать когомологии обычным образом (при котором они являются гомотопическими инвариантами), определенная так эйлерова характеристика не обладает свойством аддитивности. Например, эйлерова характеристика окружности $S^{1}$ равна 0 . Представим окружность в виде объединения точки и дополнения к ней. Эйлерова характеристика точки равна 1. Дополнение $S^{1} \backslash\{\mathrm{pt}\}$ гомеоморфно прямой $\mathbb{R}^{1}$ и гомотопически эквивалентно точке. Поэтому его эйлерова характеристика также равна 1. Тем самым $\chi\left(S^{1}\right) \neq$ $\chi(\{\mathrm{pt}\})+\chi\left(S^{1} \backslash\{\mathrm{pt}\}\right)$, т. е. эйлерова характеристика, определенная формулой (2), не аддитивна.

Для удобства дальнейшего обсуждения временно ограничимся классом пространств, которые являются локально замкнутыми объединениями клеток в конечных клеточных (CW-) комплексах (и тем самым сами являются объединениями конечного числа клеток). [Условие локальной замкнутости ("клеточного") подпространства $X$ клеточного комплекса $Z$ означает, что если клетка $a$ примыкает к клетке $b$, а клетка $b$ примыкает к клетке $c$ и при этом клетки $a$ и $c$ лежат в подпространстве $X$, то и клетка $b$ лежит в нем. Каждая индивидуальная клетка является пространством указанного класса.] Пространства этого класса алгебру множеств не образуют: объединение двух пространств, вообще говоря, не является пространством из этого класса. Алгебра множеств, порожденная 
пространствами из описанного класса, состоит из произвольных объединений клеток в конечных CW-комплексах. Для пространства $X$ указанного типа (т. е. локально замкнутого объединения клеток в конечном CW-комплексе) группы когомологий с компактными носителями $H_{\mathrm{c}}^{q}(X)$ (или, что для них то же самое, группы когомологий Бореля-Мура) - это группы когомологий $H^{q}\left(X^{*}, *\right)$ одноточечной компактификации $X^{*}$ пространства $X$ по модулю добавленной точки $*$. Определим эйлерову характеристику $\chi(X)$ пространства $X$ как

$$
\chi(X)=\sum_{q \geqslant 0}(-1)^{q} \operatorname{dim} H_{\mathrm{c}}^{q}(X) .
$$

Предположим, что $Y$ - замкнутое подпространство в $X$ (оба пространства из указанного класса, т. е. являются локально замкнутыми объединениями клеток в конечном $\mathrm{CW}$-комплексе). Из точной когомологической последовательности

$$
\cdots \rightarrow H^{q}\left(X^{*}, Y^{*}\right) \rightarrow H^{q}\left(X^{*}, *\right) \rightarrow H^{q}\left(Y^{*}, *\right) \rightarrow H^{q+1}\left(X^{*}, Y^{*}\right) \rightarrow \cdots
$$

и равенства $H^{q}\left(X^{*}, Y^{*}\right)=H^{q}\left((X \backslash Y)^{*}, *\right)$ следует, что

$$
\chi(X)=\chi(Y)+\chi(X \backslash Y) .
$$

Тем самым, эйлерова характеристика, определенная формулой (3), аддитивна (для пространств указанного типа). Для компактных пространств (конечных CW-комплексов) эйлеровы характеристики, определенные формулами (2) и (3), совпадают.

Нетрудно видеть, что эйлерова характеристика $\chi\left(\sigma^{d}\right)$ клетки $\sigma^{d}$ размерности $d$ равна $(-1)^{d}$. Эйлерова характеристика произвольного пространства $X$ из описанного класса равна альтернированной сумме количеств клеток различных размерностей в $X$. Эйлерова характеристика (3) гомотопическим инвариантом не является. Однако она является инвариантом пространства относительно гомотопической эквивалентности в категории собственных отображений (т. е. отображений, для которых прообраз компактного подпространства компактен). Нетрудно видеть, что она очевидным образом продолжается до аддитивной функции на алгебре множеств, состоящей из объединений клеток в конечных CW-комплексах.

В дальнейшем обычно мы будем рассматривать эйлерову характеристику как функцию на другой (в некотором смысле более узкой) алгебре множеств алгебре конструктивных множеств. Одна из основных причин, вынуждающая так поступать, состоит в следующем. В разумных задачах пространства редко фигурируют вместе с фиксированными клеточными разбиениями. Идея рассматривать пространства, имеющие клеточные разбиения, не реализуема из-за того, что они не образуют алгебру: объединение и пересечение допускающих клеточные разбиения пространств не обязаны иметь клеточные разбиения. Конструктивные же множества допускают клеточные разбиения и образуют алгебру множеств. Кроме того, алгебра конструктивных множеств выдерживает взятие образа: в соответствии с теоремой Шевалле образ конструктивного множества при алгебраическом отображении конструктивен. Этот факт важен, в частности, для теоремы Фубини (теорема 1 ниже). 
Проективное множество (над полем $\mathbb{C}$ комплексных чисел) - это подмножество проективного пространства $\mathbb{C P}^{N}$, определяемое набором полиномиальных (однородных) уравнений относительно проективных координат. Полуалгебраическим (или квазипроективным) множеством называется разность двух (комплексных) проективных (алгебраических) множеств. Любое проективное множество имеет клеточное разбиение, т. е. представимо в виде (конечного) CW-комплекса, а любое полуалгебраическое подмножество является локально замкнутым объединением клеток в нем (при некотором клеточном разбиении последнего): см., например, [10]. Алгебра множеств, порожденная полуалгебраическими множествами, состоит из конструктивных множеств конечных объединений полуалгебраических. Эйлерова характеристика, определенная для полуалгебраических множеств формулой (3), продолжается до аддитивной функции на алгебре конструктивных множеств.

ЗАмЕчАниЕ. В действительности, для комплексных полуалгебраических множеств эйлеровы характеристики, определенные формулами (2) и (3), совпадают. Определение, даваемое формулой (3), необходимо, если рассматривать другие алгебры множеств, например, алгебру объединений клеток в конечных CW-комплексах или алгебру вещественных конструктивных множеств.

Эйлерова характеристика мультипликативна в следующем смысле: если $p$ : $E \rightarrow B$ - локально тривиальное (в топологическом смысле) расслоение со слоем $F$, то $\chi(E)=\chi(B) \chi(F)$.

Для ряда задач удобно рассматривать эйлерову характеристику на алгебре вещественных конструктивных множеств. Полуалгебраическим множеством над полем вещественных чисел называется подмножество (вещественного) проективного пространства, задаваемое в любой аффинной карте конечным набором алгебраических уравнений и неравенств (> или $\geqslant)$. Любое полуалгебраическое множество представимо в виде (локально замкнутого) объединения клеток в конечном CW-комплексе. Вещественное конструктивное множество - это конечное объединение полуалгебраических. Как и в комплексном случае, эйлерова характеристика, определенная для вещественных полуалгебраических множеств формулой (3), продолжается до аддитивной функции на алгебре вещественных конструктивных множеств. Для вещественных полуалгебраических множеств эйлеровы характеристики, определенные формулами (2) и (3), вообще говоря, различны (см. пример выше). Для определения интеграла по отношению к эйлеровой характеристике следует пользоваться аддитивной, которая определяется формулой (3).

\section{3. Интеграл по отношению к эйлеровой характеристике}

Теперь мы готовы дать определение интеграла по отношению к эйлеровой характеристике.

Функция $\psi$ на конструктивном множестве $X$ со значениями в абелевой группе $G$ называется конструктивной, если для любого элемента $a \in G$ соответствующее множество уровня $\psi^{-1}(a)$ конструктивно. Сумма конструктивных функций конструктивна (как и их произведение в случае, если $G$ - кольцо). 
Конструктивная функция имеет конечное число различных значений. Поэтому имеет смысл следующее определение.

ОПРЕДЕЛЕНИЕ. Интеграл конструктивной функции $\psi$ по отношению $\kappa$ эйлеровой характеристике (или, как иногда говорят, по эйлеровой характеристике) по пространству $X$ - это

$$
\int_{X} \psi d \chi=\sum_{a \in G} \chi\left(\psi^{-1}(a)\right) a .
$$

Интеграл по отношению к эйлеровой характеристике обладает обычными свойствами аддитивности по отношению к области интегрирования и по отношению к подынтегральной функции. Имеет место очевидное равенство

$$
\int_{X} 1 d \chi=\chi(X)
$$

где 1 - постоянная функция на $X$ со значениями в группе $\mathbb{Z}$ целых чисел (принимающая значение 1 во всех точках пространства $X$ ).

Одним из важнейших свойств интеграла по отношению к эйлеровой характеристике, используемых в приложениях, является теорема Фубини.

ОПРедЕлЕниЕ. Отображение $f: X \rightarrow Y$ конструктивных множеств называется конструктивным, если существуют такие представления множеств $X$ и $Y$ в виде конечных объединений $X=\bigsqcup_{i \in I} X_{i}, Y=\bigsqcup_{j \in J} Y_{j}$ непересекающихся полуалгебраических множеств, что для любого $i \in I$ существует $j \in J$ такое, что $f\left(X_{i}\right) \subset Y_{j}$ и ограничение отображения $f$ на множество $X_{i}$ является алгебраическим отображением $X_{i} \rightarrow Y_{j}$.

Теорема 1. Пусть $f: X \rightarrow Y$ - конструктивное отображсение, $\psi: X \rightarrow G-$ конструктивная функция на множестве $X$ со значениями в абелевой группе $G$. Тогда функиия $\widehat{\psi}: Y \rightarrow G$, определенная формулой

$$
\widehat{\psi}(y)=\int_{f^{-1}(y)} \psi d \chi
$$

для $y \in Y$ (интеграл по слоям отображения $f)$, конструктивна и

$$
\int_{X} \psi d \chi=\int_{Y}\left(\int_{f^{-1}(y)} \psi d \chi\right) d \chi
$$

ДокАзАтЕльство. Утверждение достаточно доказать для случая, когда $X$ и $Y$ - полуалгебраические множества, а $f$ - алгебраическое отображение. Более того, вследствие аддитивности интеграла достаточно рассмотреть случай, когда функция $\psi$ на множестве $X$ постоянна (и принимает на нем значение, равное $a$; для этой редукции надо разбить множество $X$ на множества уровня функции $\psi)$. Согласно [11], в этом случае существует разбиение образа $Y$ на конечное число непересекающихся полуалгебраических множеств $Y_{j}$ такое, 
что для каждого $j$ ограничение отображения $f$ на прообраз $f^{-1}\left(Y_{j}\right)$ множества $Y_{j}$ является локально тривиальным расслоением $f^{-1}\left(Y_{j}\right) \rightarrow Y_{j}$ со слоем $f^{-1}(y) \cong F_{j}, y \in Y_{j}$. Для $y \in Y_{j}$ интеграл по слою

$$
\widehat{\psi}(y)=\int_{f^{-1}(y)} \psi d \chi
$$

постоянен и равен $\chi\left(F_{j}\right) a$. Поэтому функция $\widehat{\psi}$ конструктивна и

$$
\begin{aligned}
\int_{X} \psi d \chi & =\sum_{j} \int_{f^{-1}\left(Y_{j}\right)} \psi d \chi=\sum_{j} \int_{f^{-1}\left(Y_{j}\right)} a d \chi \\
& =\sum_{j} \chi\left(f^{-1}\left(Y_{j}\right)\right) a=\sum_{j} \chi\left(Y_{j}\right) \chi\left(F_{j}\right) a=\int_{Y} \widehat{\psi} d \chi .
\end{aligned}
$$

СЛЕДСТВИЕ. Имеет место равенство

$$
\chi(X)=\int_{Y} \chi\left(f^{-1}(y)\right) d \chi .
$$

Если групповая операция в $G$ записывается как умножение, то правая часть формулы (5) превращается в

$$
\prod_{a \in G} a^{\chi\left(\psi^{-1}(a)\right)} .
$$

В этом случае сам интеграл по отношению к эйлеровой характеристике удобно записывать в виде

$$
\int_{X} \psi^{d \chi}
$$

помещая $d \chi$ в показатель степени. Например, формула Н. А'Кампо (пример п. 4.2 ниже) записывается в виде такого интеграла от функции со значениями в мультипликативной группе рациональных функций от одной переменной со значением 1 в нуле.

\section{4. Некоторые примеры}

4.1. Пусть $S$ - замкнутая (компактная, без края) риманова поверхность (комплексное одномерное многообразие) рода $g$. Пусть $p: \widetilde{S} \rightarrow S$ - ее разветвленное накрытие порядка $k$ с $m$ точками ветвления $x_{1}, \ldots, x_{m} \in \widetilde{S}$ такими, что порядок ветвления в точке $x_{i}$ равен $r_{i}(i=1, \ldots, m)$. Формула Римана-Гурвица дает род $\tilde{g}$ поверхности $\widetilde{S}$.

Теорема 2 (Формула Римана-Гурвица). Эйлеровы характеристики поверхности $S$ и ее накрытия $\widetilde{S}$ связаны соотношением

$$
\chi(\widetilde{S})=k \chi(S)-\sum_{i=1}^{m}\left(r_{i}-1\right)
$$

$(\chi(S)=2-2 g, \chi(\widetilde{S})=2-2 \tilde{g})$. 
ДокАЗАТЕЛЬство. Имеют место равенства

$$
\begin{aligned}
\chi(\widetilde{S}) & =\int_{\widetilde{S}} 1 d \chi=\int_{S}\left(\int_{p^{-1}(x)} 1 d \chi\right) d \chi=\int_{S} \# p^{-1}(x) d \chi \\
& =k \chi(S)-\int_{S}\left(k-\# p^{-1}(x)\right) d \chi=k \chi(S)-\sum_{i=1}^{m}\left(r_{i}-1\right)
\end{aligned}
$$

[здесь $\# X-$ количество элементов в (конечном) множестве $X]$.

4.2. Известная теорема Н. А'Кампо [12] выражает дзета-функцию преобразования классической монодромии ростка голоморфной функции в терминах разрешения ростка. Пусть $f:\left(\mathbb{C}^{n}, 0\right) \rightarrow(\mathbb{C}, 0)-$ росток голоморфной функции. Обозначим через $f$ также его представителя, определенного в некоторой окрестности $U$ начала координат в $\mathbb{C}^{n}$. Пусть $D_{\rho}(0)$ - замкнутый диск радиуса $\rho$ с центром в начале координат в $\mathbb{C}, B_{\delta}(0)$ - замкнутый шар радиуса $\delta$ с центром в начале координат в $\mathbb{C}^{n}$. Для достаточно малых $0<\rho \ll \delta$ отображение

$$
f: f^{-1}\left(D_{\rho}(0) \backslash\{0\}\right) \cap B_{\delta}(0) \rightarrow D_{\rho}(0) \backslash\{0\}
$$

является локально тривиальным $\left(C^{\infty}-\right)$ расслоением - расслоением Милнора pостка $f$ : [13], [14]. Слой $V_{f}=f^{-1}(\varepsilon)(0<|\varepsilon| \leqslant \rho)$ этого расслоения называется слоем Милнора ростка $f$. Слой Милнора $V_{f}$ является комплексным многообразием с (вещественным) краем $\partial V_{f}=V_{f} \cap S_{\delta}^{2 n-1}(0)\left(S_{\delta}^{2 n-1}(0)=\partial B_{\delta}(0)-\right.$ сфера радиуса $\delta$ с центром в начале координат в $\left.\mathbb{C}^{n}\right)$. Если росток $f$ имеет изолированную критическую точку в начале координат, то слой Милнора $V_{f}$ гомотопически эквивалентен букету сфер размерности $n-1$ : [13]. Пусть $h_{f}: V_{f} \rightarrow V_{f}$ - преобразование монодромии расслоения Милнора, соответствующее петле $\sigma(t)=\varepsilon e^{2 \pi i t}, 0 \leqslant t \leqslant 1,-$ преобразование классической монодромии ростка $f$.

ОПРЕДЕЛЕниЕ. Дзета-функцией $\zeta_{h}(t)$ преобразования $h: X \rightarrow X$ пространства $X$ (конечномерного, с конечномерными группами когомологий) называется рациональная функция

$$
\zeta_{h}(t)=\prod_{q \geqslant 0}\left\{\operatorname{det}\left[\operatorname{id}-\left.t h^{*}\right|_{H_{\mathrm{c}}^{q}(X ; \mathbb{R})}\right]\right\}^{(-1)^{q+1}} .
$$

Степень дзета-функции $\zeta_{h}(t)$ (степень числителя минус степень знаменателя) равна минус эйлеровой характеристике пространства $X$.

Дзета-функция $\zeta_{h}(t)$ преобразования $h: X \rightarrow X$ обладает следующим свойством мультипликативности (аналогом свойства (4) аддитивности эйлеровой характеристики). Пусть $Y$ - замкнутое подмножество в $X$ и $h(Y) \subset Y$, $h(X \backslash Y) \subset X \backslash Y$. Тогда $\zeta_{h}(t)=\zeta_{h_{\mid Y}}(t) \zeta_{h_{\mid X \backslash Y}}(t)$.

Дзета-функция $\zeta_{f}(t)$ преобразования $h_{f}: V_{f} \rightarrow V_{f}$ классической монодромии голоморфного ростка $f:\left(\mathbb{C}^{n}, 0\right) \rightarrow(\mathbb{C}, 0)$ называется дзета-функиией (монодромии) ростка $f$.

ЗАмЕчАНИЕ. Иногда в определении дзета-функции преобразования вместо $q+1$ в показатели степени ставится $q$. Дзета-функция, определенная таким образом, является обратной к (6). Используемый здесь выбор показателя степени 
объясняется связью дзета-функции неприводимого ростка функции двух переменных с многочленом Александера соответствующего алгебраического узла $f^{-1}(0) \cap S_{\delta}^{3}(0) \subset S_{\delta}^{3}(0)$, используемой в разделе 6 .

ВАжный ПРИмеР. Пусть $f\left(x_{1}, \ldots, x_{n}\right)=x_{1}^{m}, m>0$. Слой Милнора $V_{f}$ ростка $f$ состоит из $m$ стягиваемых компонент. Преобразование классической монодромии $h_{f}$ переставляет эти компоненты циклически. Отсюда следует, что $\zeta_{f}(t)=\left(1-t^{m}\right)^{-1}$. Пусть $n \geqslant 2, f\left(x_{1}, \ldots, x_{n}\right)=x_{1}^{m_{1}} \cdots x_{s}^{m_{s}}$, где $s \geqslant 2$ и $m_{i}-$ положительные целые числа. Нетрудно показать, что $\zeta_{f}(t)=1$. Это можно вывести, например, из того, что на слое Милнора ростка $f$ можно построить действие окружности без неподвижных точек, коммутирующее с преобразованием монодромии $h_{f}$.

Пусть $\pi:(X, D) \rightarrow\left(\mathbb{C}^{n}, 0\right)$ - разрешение ростка $f$. Это означает, что:

1) $X$ - гладкое комплексное многообразие (размерности $n$ );

2) $\pi$ - собственное (прообраз компакта компактен) голоморфное отображение, являющееся изоморфизмом вне $f^{-1}(0) \subset\left(\mathbb{C}^{n}, 0\right)$;

$3)$ в окрестности любой точки $x \in(f \circ \pi)^{-1}(0) \subset X$ на многообразии $X$ существуют локальные координаты $z_{1}, \ldots, z_{n}$, центрированные в точке $x$ и такие, что $f \circ \pi\left(z_{1}, \ldots, z_{n}\right)=z_{1}^{m_{1}} \cdots z_{n}^{m_{n}}$, где $m_{1}, \ldots, m_{n}$ - неотрицательные целые числа.

Последнее условие означает, что $(f \circ \pi)^{-1}(0)$ - дивизор с нормальными пересечениями на $X$. Для $m \geqslant 1$ обозначим через $S_{m}$ множество точек $x \in D=$ $\pi^{-1}(0)$, в окрестности которых функция $f \circ \pi$ представима в виде $z_{1}^{m}$ (т. е. только одно из чисел $m_{i}$ отлично от нуля и равно $m$ ).

Теорема 3 (теорема А'Кампо). Дзета-функиия ростка $f$ задается формулой

$$
\zeta_{f}(t)=\prod_{m \geqslant 1}\left(1-t^{m}\right)^{-\chi\left(S_{m}\right)} .
$$

Это утверждение можно вывести, например, из "Важного примера" с использованием указанного выше свойства мультипликативности дзета-функции преобразования. Из теоремы А'Кампо вытекает, что для ростка $f$ с изолированной критической точкой в начале координат действие преобразования классической монодромии на группах когомологий слоя Милнора унипотентно, т. е. существуют такие положительные целые числа $q$ и $N$, что $\left(\left(h^{*}\right)^{q}-\mathrm{id}\right)^{N}=0$. (В действительности это выполняется для $N$, равного количеству переменных $n$.) Доказательство этого свойства для любого ростка функции (не обязательно с изолированной критической точкой в начале координат) может быть найдено, например, в [15].

Для $x \in D$ обозначим через $\zeta_{x}(t)$ дзета-функцию монодромии ростка в точке $x$ поднятия $f \circ \pi$ функции $f$ на пространство $X$ разрешения. Будем рассматривать $\zeta_{x}(t)$ как функцию на исключительном дивизоре $D$ со значениями в (мультипликативной) группе рациональных функций переменной $t$, принимающих значение 1 в нуле. Из "Важного примера" выше следует, что формула (7) может быть записана в виде

$$
\zeta_{f}(t)=\int_{D}\left(\zeta_{x}(t)\right)^{d \chi} .
$$


4.3. Формула (8) имеет гораздо более широкую область приложения. Предположим, что $\pi:(X, D) \rightarrow\left(\mathbb{C}^{n}, 0\right)$ - собственное отображение гладкого многообразия $X$, являющееся изоморфизмом вне нулевого множества уровня $f^{-1}(0) \subset\left(\mathbb{C}^{n}, 0\right)$, но разрешением ростка $f$, вообще говоря, не являющееся (т. е. $(f \circ \pi)^{-1}(0)$ не является дивизором с нормальными пересечениями на $\left.X\right)$. Пусть, как и выше, $\zeta_{x}(t)$ - дзета-функция монодромии ростка функции $f \circ \pi$ в точке $x \in D=\pi^{-1}(0)$.

Теорема 4 [16]. Имеет место равенство

$$
\zeta_{f}(t)=\int_{D}\left(\zeta_{x}(t)\right)^{d \chi}
$$

Соотношения, аналогичные (9), имеют место и для ряда других (в частности, нелокальных) ситуаций.

Пусть $P \in \mathbb{C}\left[x_{1}, \ldots, x_{n}\right]$ - многочлен от $n$ переменных. Он определяет отображение $P: \mathbb{C}^{n} \rightarrow \mathbb{C}$, являющееся локально тривиальным $\left(C^{\infty}-\right)$ расслоением вне конечного множества $\Sigma \subset \mathbb{C}$ - бифуркационного множества полиномиального отображения $P$ (см. [11], [17]). Обозначим через $V_{P}$ слой этого расслоения - множество уровня многочлена $P$ общего положения. Пусть $\sigma(t)=R e^{2 \pi i t}$, $0 \leqslant t \leqslant 1,-$ петля достаточно большого радиуса $R$ (так что все точки бифуркационного множества $\Sigma$ лежат внутри этой петли). Преобразование монодромии $h_{P}^{\infty}: V_{P} \rightarrow V_{P}$ описанного расслоения, соответствующее этой петле, называется преобразованием монодромии многочлена $P$ на бесконечности. Дзета-функция $\zeta_{P}^{\infty}(t)$ преобразования монодромии $h_{P}^{\infty}$ называется дзета-функиией (монодромии) многочлена $P$ на бесконечности.

Полиномиальная функция $P: \mathbb{C}^{n} \rightarrow \mathbb{C}$ является мероморфной функцией на проективном замыкании $\mathbb{C P}^{n}$ пространства $\mathbb{C}^{n}$. Пусть $\Phi=\frac{F}{G}-$ мероморфная функция на компактном комплексном многообразии $M^{n}$ размерности $n$ $(F$ и $G$ - голоморфные сечения некоторого линейного расслоения $L$ на $M)$. Отображение $\Phi$ из дополнения $M \backslash\{F=G=0\}$ к множеству $\{F=G=0\}$ неопределенности функции $\Phi$ в проективную комплексную прямую $\mathbb{C P}^{1}$ является локально тривиальным расслоением вне конечного множества в $\mathbb{C P}^{1}$ (бифуркационного множества). Для фиксированного значения $c \in \mathbb{C P}^{1}$ пусть $h_{\Phi}^{c}-$ преобразование монодромии описанного расслоения, соответствующее обходу по маленькой окружности вокруг значения $c$. Его дзета-функция $\zeta_{\Phi}^{c}(t)$ называется дзета-функиией монодромии мероморфной функции $\Phi$ вокруг значения $c$.

Пусть $\varphi=\frac{f}{g}$ - росток мероморфной функции на $\left(\mathbb{C}^{n}, 0\right)(f$ и $g$ - ростки голоморфных функций $\left.\left(\mathbb{C}^{n}, 0\right) \rightarrow(\mathbb{C}, 0)\right)$. Два ростка $\varphi=\frac{f}{g}$ и $\varphi^{\prime}=\frac{f^{\prime}}{g^{\prime}}$ считаются равными, если существует голоморфный росток $u:\left(\mathbb{C}^{n}, 0\right) \rightarrow(\mathbb{C}, 0)$ такой, что $u(0) \neq 0, f^{\prime}=u f, g^{\prime}=u g$. (Таким образом, например, $\frac{x}{y} \neq \frac{x^{2}}{x y}$. Это позволяет рассматривать мероморфные ростки с $(n-1)$-мерными множествами неопределенности.)

Отображение $\varphi: B_{\rho}(0) \backslash\{f=g=0\} \rightarrow \mathbb{C P}^{1}\left(B_{\rho}(0)\right.$ - замкнутый шар радиуса $\rho>0$ с центром в начале координат в $\left.\mathbb{C}^{n}\right)$, вообще говоря, не является 
локально тривиальным расслоением вне конечного подмножества в $\mathbb{C P}^{1}$. Однако для фиксированного $c \in \mathbb{C P}^{1}$ при достаточно малом $\rho$ отображение $\varphi$ является локально тривиальным расслоением над проколотой окрестностью точки $c$ : [18], [19]. Слой

$$
M_{\varphi}^{c^{\prime}}=\left\{x \in B_{\rho}(0): \varphi(x)=\frac{f(x)}{g(x)}=c^{\prime}\right\}
$$

этого расслоения $\left(0<\operatorname{dist}\left(c, c^{\prime}\right) \ll \rho\right)$ называется слоем Милнора ростка $\varphi$, соответствующим значению $c$. Преобразование монодромии этого расслоения, соответствующее обходу по малой окружности вокруг $c$, и его дзета-функция $\zeta_{\varphi}^{c}(t)$ называются преобразованием классической монодромии и дзета-функиией монодромии мероморфного ростка $\varphi$, соответствующими значению $c$.

Дзета-функция монодромии мероморфной функции (на замкнутом комплексном многообразии, например, на проективном пространстве $\mathbb{C P}^{n}$ ) равна интегралу по отношению к эйлеровой характеристике дзета-функций локальных

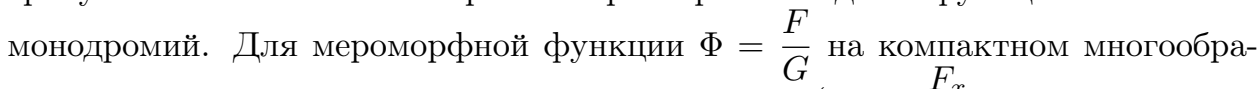
зии $M^{n}$ обозначим через $\Phi_{x}$ ее росток в точке $x\left(\Phi_{x}=\frac{F_{x}}{G_{x}}\right.$, где в некоторой локальной тривиализации линейного расслоения $L$ ростки его сечений $F_{x}$ и $G_{x}$ являются ростками голоморфных функций), а через $\zeta_{\Phi, x}^{c}(t)$ - дзета-функцию монодромии этого ростка, соответствующую значению $c$.

Tеорема 5 [20], [19]. Имеет место равенство

$$
\zeta_{\Phi}^{c}(t)=\int_{M^{n}}\left(\zeta_{\Phi, x}^{c}(t)\right)^{d \chi}
$$

Доказательство выводится, например, из аналога теоремы A'Кампо для мероморфных функций.

ЗАмЕчАниЕ. Легко видеть, что для точки $x$, не принадлежащей множеству неопределенности $\{F=G=0\}$ и такой, что $\Psi(x) \neq c$, выполнено равенство

$$
\zeta_{\Phi, x}^{c}(t)=1
$$

Поэтому множеством интегрирования в формуле (10) можно считать объединение $\{F=G=0\} \cup \Psi^{-1}(c)$ множества неопределенности и множества уровня функции $\Psi$, соответствующего значению $c$. В аналоге формулы (10) для дзета-функции монодромии многочлена на бесконечности [21] интегрирование производится по бесконечно удаленной гиперплоскости.

Для полиномиальных функций на аффинном пространстве $\mathbb{C}^{n}$ аналог теоремы 5 был сформулирован и использовался в [21]. Другим примером подобной локализации является представление дзета-функции (однопараметрической) деформации полиномиальной функции на аффинном пространстве $\mathbb{C}^{n}$ в виде интеграла по отношению к эйлеровой характеристике от локальных данных (дзета-функций деформаций мероморфных ростков) в проективном пополнении $\mathbb{C P}^{n}$ пространства $\mathbb{C}^{n}[22]$. 


\section{5. Ряды Пуанкаре фильтраций и интегралы по эйлеровой характеристике}

Интегрировать по отношению к эйлеровой характеристике можно не только по конечномерным (конструктивным) пространствам, но и по некоторым бесконечномерным. Для этого надо выделить некоторую алгебру подмножеств такого пространства, для элементов которой определить понятие эйлеровой характеристики. В частности, может быть определен интеграл по отношению к эйлеровой характеристике по кольцу $\mathscr{O}_{V, 0}$ ростков комплексно аналитических функций на ростке $(V, 0)$ (вообще говоря, особого) комплексно аналитического пространства и по его проективизации $\mathbb{P} \mathscr{O}_{V, 0}[23],[24]$. Для приложений в этом и следующем разделах нам потребуется именно интеграл по проективизации $\mathbb{P} \mathscr{O}_{V, 0}$ кольца функций. Поэтому мы опишем конструкцию для этого случая. (Интеграл по кольцу $\mathscr{O}_{V, 0}$ определяется аналогично.)

Для векторного пространства $E$ (конечномерного или бесконечномерного) обозначим через $\mathbb{P} E$ его проективизацию (для бесконечномерного пространства $E$ проективизацию $\mathbb{P} E$ здесь можно рассматривать просто как множество), через $\mathbb{P}^{*} E$ - объединение $\mathbb{P} E \cup\{*\}$ проективизации $\mathbb{P} E$ и дополнительной точки $\{*\}$. (Можно представлять себе, что $\mathbb{P}^{*} E-$ это множество орбит естественного $\mathbb{C}^{*}$-действия на $E,\{*\}$ - орбита, состоящая из начала координат.)

Пусть $\mathfrak{m}$ - максимальный идеал в кольце $\mathscr{O}_{V, 0}$. Обозначим через $d_{k}$ размерность факторпространства $\mathscr{O}_{V, 0} / \mathfrak{m}^{k+1}$. Пространство $J_{V, 0}^{k} k$-струй функций на ростке $(V, 0)$ - это фактор $\mathscr{O}_{V, 0} / \mathfrak{m}^{k+1}$ по $(k+1)$-й степени максимального идеала $\left(d_{k}=\operatorname{dim} J_{V, 0}^{k}\right)$. Имеются естественные отображения ("усечения") $\pi_{k}: \mathbb{P} \mathscr{O}_{V, 0} \rightarrow \mathbb{P}^{*} J_{V, 0}^{k}$ и $\pi_{k, \ell}: \mathbb{P} J_{V, 0}^{k} \rightarrow \mathbb{P}^{*} J_{V, 0}^{\ell}$ для $k \geqslant \ell$. Над $\mathbb{P} J_{V, 0}^{\ell} \subset \mathbb{P}^{*} J_{V, 0}^{\ell}$ отображение $\pi_{k, \ell}$ является локально тривиальным (в том числе и в топологии Зариского) расслоением, слоем которого является аффинное пространство размерности $d_{k}-d_{\ell}$.

ОПРЕДЕЛЕНИЕ. Подмножество $X \subset \mathbb{P} \mathscr{O}_{V, 0}$ называется цилиндрическим, если существуют $k \geqslant 0$ и конструктивное подмножество $Y \subset \mathbb{P} J_{V, 0}^{k}\left(\subset \mathbb{P}^{*} J_{V, 0}^{k}\right)$ такие, что $X=\pi_{k}^{-1}(Y)$.

Цилиндрические подмножества проективизации $\mathbb{P} \mathscr{O}_{V, 0}$ образуют алгебру множеств.

ОПРедЕЛЕНИЕ. Эйлерова характеристика $\chi(X)$ цилиндрического подмножества $X \subset \mathbb{P} \mathscr{O}_{V, 0}$ такого, что $X=\pi_{k}^{-1}(Y), Y \subset \mathbb{P} J_{V, 0}^{k}$, определяется как эйлерова характеристика $\chi(Y)$ соответствующего (конструктивного) множества $Y$.

Корректность этого определения вытекает из того, что если цилиндрическое подмножество $X$ также равно $\pi_{k^{\prime}}^{-1}\left(Y^{\prime}\right)$ для $k^{\prime}>k, Y^{\prime} \subset \mathbb{P} J_{V, 0}^{k^{\prime}}$, то $Y^{\prime}$ является локально тривиальным расслоением над $Y$, слоем которого является (комплексное) аффинное пространство (см. выше). Поскольку эйлерова характеристика комплексного аффинного пространства равна 1, эйлеровы характеристики множеств $Y$ и $Y^{\prime}$ совпадают.

Пусть $\left\{F_{i}\right\}$,

$$
F_{0}=\mathscr{O}_{V, 0} \supset F_{1} \supset F_{2} \supset \cdots,
$$


- (убывающая) фильтрация на кольце $\mathscr{O}_{V, 0}, F_{i}$ - векторные подпространства. Обозначим через $v$ функцию на кольце $\mathscr{O}_{V, 0}$ со значениями в $\mathbb{Z}_{\geqslant 0} \cup\{+\infty\}$, определенную формулой $v(f)=\sup \left\{i: f \in F_{i}\right\}$. Функция $v$ удовлетворяет условию $v(c f)=v(f)$ для ненулевого $c \in \mathbb{C}$ и условию $v(f+g) \geqslant \min (v(f), v(g))$. Функцию $v: \mathscr{O}_{V, 0} \rightarrow \mathbb{Z}_{\geqslant 0} \cup\{+\infty\}$, удовлетворяющую этим условиям, мы будем называть функцией порядка. (Если функция порядка удовлетворяет еще и условию $v(f g)=v(f)+v(g)$, то она является нормированием.) Фильтрация $\left\{F_{i}\right\}$ определяется соответствующей функцией порядка $v$ :

$$
F_{i}=\left\{f \in \mathscr{O}_{V, 0}: v(f) \geqslant i\right\}
$$

Будем говорить, что фильтрация $\left\{F_{i}\right\}$ конечно определена, если для любого $i$ существует такое $N$, что $\mathfrak{m}^{N} \subset F_{i}$. Если фильтрация $\left\{F_{i}\right\}$ конечно определена, то все факторпространства $F_{i} / F_{i+1}, i \geqslant 0$, конечномерны. Это означает, что для фильтрации $\left\{F_{i}\right\}$ (заданной функцией порядка $v$ ) определен ее $р я \partial$ Пуанкаре

$$
P_{\{v\}}(t)=\sum_{i=0}^{\infty} \operatorname{dim}\left(F_{i} / F_{i+1}\right) t^{i} .
$$

Будем рассматривать моном $t^{v}=t^{v(g)}$ как функцию на проективизации $\mathbb{P} \mathscr{O}_{V, 0}$ кольца $\mathscr{O}_{V, 0}$ со значениями в абелевой группе (по сложению) $\mathbb{Z}[[t]]$ степенных рядов от переменной $t$ с целыми коэффициентами. При этом $t^{+\infty}$ считаем равным нулю. Имеют место следующие очевидные соотношения.

(а) Если $E$ - конечномерное (комплексное) векторное пространство, то $\operatorname{dim} E=\chi(\mathbb{P} E)$.

(b) Если $E \supset E^{\prime}$ - конечномерные векторные пространства, то $\operatorname{dim}\left(E / E^{\prime}\right)=$ $\chi\left(\mathbb{P} E \backslash \mathbb{P} E^{\prime}\right)$.

C учетом этого следующее утверждение вытекает непосредственно из определения интеграла по отношению к эйлеровой характеристике.

ПредЛОжениЕ 1. Для конечно определенной фильтрации $\left\{F_{i}\right\}$ на кольце $\mathscr{O}_{V, 0}$ имеет место соотношение

$$
P_{\{v\}}(t)=\int_{\mathbb{P} \mathscr{O}_{V, 0}} t^{v(g)} d \chi
$$

ДокАзАТЕЛЬСтво. Имеют место равенства

$$
\int_{\mathbb{P} \mathscr{O}_{V, 0}} t^{v(g)} d \chi=\sum_{i=0}^{\infty} \chi\left(\mathbb{P} F_{i} \backslash \mathbb{P} F_{i+1}\right) t^{i}=\sum_{i=0}^{\infty} \operatorname{dim}\left(F_{i} / F_{i+1}\right) t^{i}=P_{\{v\}}(t) .
$$

Перейдем теперь к мультииндексным фильтрациям. В литературе это понятие не имеет точно зафиксированного определения. Так, под $r$-индексной фильтрацией кольца $\mathscr{O}_{V, 0}$ можно понимать такое семейство его подпространств $\{J(\underline{v})\}$, занумерованных индексом $\underline{v}=\left(v_{1}, \ldots, v_{r}\right) \in \mathbb{Z}_{\geqslant 0}^{r}$, что $J\left(\underline{v}^{\prime}\right) \subset J(\underline{v})$ при $\underline{v}^{\prime} \geqslant \underline{v}$ (последнее означает, что $v_{i}^{\prime} \geqslant v_{i}$ для всех $i=1, \ldots, r$, где $\underline{v}^{\prime}=$ $\left.\left(v_{1}^{\prime}, \ldots, v_{r}^{\prime}\right)\right)$. Мультииндексные фильтрации в этом смысле рассматриваются, например, в [25]. Мы будем рассматривать более узкий класс мультииндексных фильтраций. 
ОПРЕДЕЛЕНИЕ. Мулътииндексной ( $r$-индексной) фильтрацией на кольце $\mathscr{O}_{V, 0}$ называется семейство подпространств $\{J(\underline{v})\}$ пространства $\mathscr{O}_{V, 0}$ (занумерованных индексом $\left.\underline{v}=\left(v_{1}, \ldots, v_{r}\right) \in \mathbb{Z}_{\geqslant 0}^{r}\right)$, которое определяется набором из $r$ функций порядка $v_{i}: \mathscr{O}_{V, 0} \rightarrow \mathbb{Z}_{\geqslant 0} \cup\{+\infty\} \quad(i=1, \ldots, r)$ :

$$
J(\underline{v})=\left\{f \in \mathscr{O}_{V, 0}: v_{i}(f) \geqslant v_{i} \text { для } i=1, \ldots, r\right\} .
$$

ЗАмЕчАниЕ. В терминах самого семейства $\{J(\underline{v})\}$ это определение означает, что $J(\underline{v}) \cap J\left(\underline{v}^{\prime}\right)=J\left(\max \left(\underline{v}, \underline{v}^{\prime}\right)\right)$, где для $\underline{v}=\left(v_{1}, \ldots, v_{r}\right), \underline{v}^{\prime}=\left(v_{1}^{\prime}, \ldots, v_{r}^{\prime}\right)$ точка $\max \left(\underline{v}, \underline{v}^{\prime}\right)$ определяется как $\left(\max \left(v_{1}, v_{1}^{\prime}\right), \ldots, \max \left(v_{r}, v_{r}^{\prime}\right)\right)$.

Примеры естественных мультииндексных фильтраций можно найти в разделе 6 .

Определение ряда Пуанкаре мультииндексной фильтрации не является прямолинейным обобщением соответствующего определения (11) для одноиндексной фильтрации. Изначально определение ряда Пуанкаре было дано в [26] в терминах подпространств $J(\underline{v})$ : см. ниже. Удобнее определить его через обобщение формулы (12).

Предположим, что фильтрация $\{J(\underline{v})\}$ (определенная функциями порядка $\left.v_{1}, \ldots, v_{r}\right)$ является конечно определенной, т. е. для любого $\underline{v} \in \mathbb{Z}_{\geqslant 0}^{r}$ существует такое $N \in \mathbb{Z}_{\geqslant 0}$, что $J(\underline{v}) \supset \mathfrak{m}^{N}$. Пусть $\underline{v}-$ функция на кольце $\mathscr{O}_{V, 0}$ со значениями в $\left(\mathbb{Z}_{\geqslant 0} \cup\{+\infty\}\right)^{r}$, определенная тем, что

$$
\underline{v}(f)=\left(v_{1}(f), \ldots, v_{r}(f)\right) .
$$

Будем рассматривать моном $\underline{t} \underline{v}=\underline{t} \underline{v}(g)$ как функцию на проективизации $\mathbb{P} \mathscr{O}_{V, 0}$ кольца (векторного пространства) $\mathscr{O}_{V, 0}$ со значениями в абелевой группе (по сложению) $\mathbb{Z}\left[\left[t_{1}, \ldots, t_{r}\right]\right]$ степенных рядов от переменных $t_{1}, \ldots, t_{r}$ с целыми коэффициентами. При этом $t_{i}^{+\infty}$ следует понимать равным нулю.

ОПРедЕЛЕНиЕ. Рядом Пуанкаре мультииндексной фильтрации $\{J(\underline{v})\}$, определенной функциями порядка $v_{1}, \ldots, v_{r}$, называется степенной ряд от $r$ переменных $t_{1}, \ldots, t_{r}$, определяемый формулой

$$
P_{\left\{v_{i}\right\}}\left(t_{1}, \ldots, t_{r}\right)=\int_{\mathbb{P} \mathscr{O}_{V, 0}} \underline{t}^{\underline{v}(g)} d \chi,
$$

где $\underline{t}=\left(t_{1}, \ldots, t_{r}\right), \underline{t} \underline{v}=t_{1}^{v_{1}} \cdots t_{r}^{v_{r}}$.

Исходное определение ряда Пуанкаре мультииндексной фильтрации $\{J(\underline{v})\}$ в [26] в терминах подпространств $J(\underline{v})$ выглядит следующим образом. Определим подпространства $J(\underline{v})$ для всех $\underline{v} \in \mathbb{Z}^{r}$ (не только для тех из них, которые имеют неотрицательные компоненты) формулой (13). Обозначим через 1 элемент $(1, \ldots, 1) \in \mathbb{Z}^{r}$.

Пусть

$$
\mathscr{L}\left(t_{1}, \ldots, t_{r}\right):=\sum_{\underline{v} \in \mathbb{Z}^{r}} \operatorname{dim}(J(\underline{v}) / J(\underline{v}+\underline{1})) \underline{t}^{\underline{v}} .
$$

Ряд $\mathscr{L}\left(t_{1}, \ldots, t_{r}\right)$ от переменных $t_{1}, \ldots, t_{r}$, вообще говоря, не является степенным: он может содержать с ненулевыми коэффициентами мономы с отрицательными показателями степеней некоторых переменных (при $r \geqslant 2$ ). Однако 
после умножения на $\prod_{i=1}^{r}\left(t_{i}-1\right)$ все мономы с отрицательными степенями переменных исчезают и

$$
\mathscr{L}\left(t_{1}, \ldots, t_{r}\right) \prod_{i=1}^{r}\left(t_{i}-1\right) \in \mathbb{Z}\left[\left[t_{1}, \ldots, t_{r}\right]\right],
$$

т. е. является степенным рядом от переменных $t_{1}, \ldots, t_{r}$.

ПредложениЕ 2. Ряд Пуанкаре филътрации связан с рядом $\mathscr{L}\left(t_{1}, \ldots, t_{r}\right)$ равенством

$$
P_{\left\{v_{i}\right\}}\left(t_{1}, \ldots, t_{r}\right)=\frac{1}{t_{1} \cdots t_{r}-1} \mathscr{L}\left(t_{1}, \ldots, t_{r}\right) \prod_{i=1}^{r}\left(t_{i}-1\right) .
$$

ДокАЗАТЕЛьСтво. Для подмножества $I \subset I_{0}=\{1,2, \ldots, r\}$ обозначим через \# $I$ количество элементов в $I$, а через $\underline{1}_{I}-$ элемент полугруппы $\mathbb{Z}_{\geqslant 0}^{r}, i$-я компонента которого равна 1 (соответственно 0), если $i \in I$ (соответственно если $i \notin I)$. В частности, $\underline{1}_{I_{0}}=\underline{1}$. Имеют место следующие равенства:

$$
\begin{aligned}
P_{\left\{v_{i}\right\}}(\underline{t})= & \int_{\mathbb{P} \mathscr{O}_{V, 0}} \underline{t}^{\underline{v}(g)} d \chi=\sum_{\underline{v} \in \mathbb{Z}^{r}} \chi\left(\mathbb{P} J(\underline{v}) \backslash \bigcup_{i=1}^{r} \mathbb{P} J\left(\underline{v}+\underline{1}_{\{i\}}\right)\right) \underline{t}^{\underline{v}} \\
= & \sum_{\underline{v} \in \mathbb{Z}^{r}} \chi\left((\mathbb{P} J(\underline{v}) \backslash \mathbb{P} J(\underline{v}+\underline{1})) \backslash \bigcup_{i=1}^{r}\left(\mathbb{P} J\left(\underline{v}+\underline{1}_{\{i\}}\right) \backslash \mathbb{P} J(\underline{v}+\underline{1})\right)\right) \underline{t}^{\underline{v}} \\
= & \sum_{\underline{v} \in \mathbb{Z}^{r}}\left(\sum_{I \subset I_{0}}(-1)^{\# I} \chi\left(\mathbb{P} J\left(\underline{v}+\underline{1}_{I}\right) \backslash \mathbb{P} J(\underline{v}+\underline{1})\right)\right) \underline{t}^{\underline{v}} \\
= & \sum_{\underline{v} \in \mathbb{Z}^{r}}\left(\sum_{I \subset I_{0}}(-1)^{\# I} \operatorname{dim}\left(J\left(\underline{v}+\underline{1}_{I}\right) / J(\underline{v}+\underline{1})\right)\right) \underline{t}^{\underline{v}} .
\end{aligned}
$$

Поэтому

$$
\begin{aligned}
& \left(t_{1} \cdots t_{r}-1\right) P_{\left\{v_{i}\right\}}(\underline{t}) \\
& =\sum_{\underline{v} \in \mathbb{Z}^{r}}\left(\sum_{I \subset I_{0}}(-1)^{\# I}\left(\operatorname{dim}\left(J\left(\underline{v}+\underline{1}_{I}-\underline{1}\right) / J(\underline{v})\right)-\operatorname{dim}\left(J\left(\underline{v}+\underline{1}_{I}\right) / J(\underline{v}+\underline{1})\right)\right)\right) \underline{t}^{\underline{v}} \\
& =\sum_{\underline{v} \in \mathbb{Z}^{r}}\left(\sum_{I \subset I_{0}}(-1)^{\# I}\left(\operatorname{dim}\left(J\left(\underline{v}+\underline{1}_{I}-\underline{1}\right) / J\left(\underline{v}+\underline{1}_{I}\right)\right)-\operatorname{dim}(J(\underline{v}) / J(\underline{v}+\underline{1}))\right)\right) \underline{t}^{\underline{v}} \\
& =\sum_{\underline{v} \in \mathbb{Z}^{r}}\left(\sum_{I \subset I_{0}}(-1)^{\# I} \operatorname{dim}\left(J\left(\underline{v}+\underline{1}_{I}-\underline{1}\right) / J\left(\underline{v}+\underline{1}_{I}\right)\right)\right) \underline{t} \underline{v} \\
& =\prod_{i=1}^{r}\left(t_{i}-1\right) \cdot \sum_{\underline{v} \in \mathbb{Z}^{r}} \operatorname{dim}(J(\underline{v}) / J(\underline{v}+\underline{1})) \underline{t}^{\underline{v}}=\prod_{i=1}^{r}\left(t_{i}-1\right) \cdot \mathscr{L}(\underline{t}) .
\end{aligned}
$$

В приведенном доказательстве возникает следующее эквивалентное определение ряда Пуанкаре:

$$
P_{\left\{v_{i}\right\}}(\underline{t})=\sum_{\underline{v} \in \mathbb{Z}_{\geqslant 0}^{r}}\left(\sum_{I \subset I_{0}}(-1)^{\# I} \operatorname{dim}\left(J\left(\underline{v}+\underline{1}_{I}\right) / J(\underline{v}+\underline{1})\right)\right) \underline{t}^{\underline{v}} .
$$


Это определение удобно для обобщения понятия ряда Пуанкаре на фильтрации, определенные более общими нормированиями или функциями порядка со значениями в упорядоченных группах, отличных от группы целых чисел $\mathbb{Z}:$ [27].

\section{6. Вычисление рядов Пуанкаре через интегралы по эйлеровой характеристике}

В качестве примера вычисления с помощью интегрирования по эйлеровой характеристике опишем вычисление ряда Пуанкаре фильтрации на кольце $\mathscr{O}_{\mathbb{C}^{2}, 0}$ ростков функций от двух переменных, определенной компонентами ростка плоской кривой: [28], [24].

Пусть $(C, 0) \subset\left(\mathbb{C}^{2}, 0\right)$ - росток плоской кривой, задаваемый уравнением $f=0, f \in \mathscr{O}_{\mathbb{C}^{2}, 0}, C=\bigcup_{i=1}^{r} C_{i}-$ его представление в виде объединения неприводимых компонент, $f=\prod_{i=1}^{r} f_{i}$, где $f_{i}=0$ - уравнение компоненты $C_{i}$. Мы предполагаем, что росток $(C, 0)$ - приведенный, т. е. $C_{i} \neq C_{j}$ при $i \neq j$, $f_{i}$ - неприводимые ростки функций из $\mathscr{O}_{\mathbb{C}^{2}, 0}$. Пусть $\varphi_{i}:(\mathbb{C}, 0) \rightarrow\left(\mathbb{C}^{2}, 0\right)-$ параметризация (униформизация) компоненты $C_{i}$ кривой $C$, т.е. росток голоморфного отображения такой, что $\operatorname{Im} \varphi_{i}=C_{i}$ и $\varphi_{i}$ является изоморфизмом между $\mathbb{C}$ и $C_{i}$ вне начала координат. Для ростка $g \in \mathscr{O}_{\mathbb{C}^{2}, 0}$ обозначим через $v_{i}(g)$ порядок нуля функции $g \circ \varphi_{i}$ в начале координат, т. е. степень начального члена в степенном разложении ростка функции $g \circ \varphi_{i}:(\mathbb{C}, 0) \rightarrow \mathbb{C}$, $g \circ \varphi_{i}(\tau)=a \tau^{v_{i}(g)}+($ члены более высокой степени $), a \neq 0$. Если $g \circ \varphi_{i}(\tau) \equiv 0$, то $v_{i}(g):=+\infty$. Функции $v_{i}, i=1, \ldots, r$, являются нормированиями на кольце $\mathscr{O}_{\mathbb{C}^{2}, 0}$ и определяют мультииндексную фильтрацию на этом кольце:

$$
J(\underline{v})=\left\{g \in \mathscr{O}_{\mathbb{C}^{2}, 0}: \underline{v}(g) \geqslant \underline{v}\right\}
$$

где

$$
\underline{v}:=\left(v_{1}, \ldots, v_{r}\right) \in \mathbb{Z}^{r} \quad \text { и } \quad \underline{v}(g):=\left(v_{1}(g), \ldots, v_{r}(g)\right) .
$$

Пусть $\pi:(X, D) \rightarrow\left(\mathbb{C}^{2}, 0\right)$ - разрешение особенности кривой $C$, т. е. собственное (прообраз компакта компактен) голоморфное отображение неособой поверхности $X$ в комплексную плоскость $\mathbb{C}^{2}$ (определенное над некоторой окрестностью начала координат в $\left.\mathbb{C}^{2}\right)$, являющееся изоморфизмом вне исключительного дивизора $D=\pi^{-1}(0)$ и такое, что полный прообраз $\pi^{-1}(C)$ кривой $C-$ дивизор с нормальными пересечениями на $X$ (а следовательно, и исключительный дивизор $D$ является дивизором с нормальными пересечениями). Такое разрешение может быть построено последовательностью $\sigma$-процессов в особых точках. Пусть $D=\bigcup_{\sigma \in \Gamma} E_{\sigma}-$ представление исключительного дивизора $D$ в виде объединения неприводимых компонент. Каждая компонента $E_{\sigma}$ изоморфна комплексной проективной прямой $\mathbb{C P}^{1}$. Для $\sigma \in \Gamma$ обозначим через $m_{i}^{\sigma}$ порядок нуля поднятия $f_{i}$ ○ $\pi$ функции $f_{i}$ вдоль компоненты $E_{\sigma}$ исключительного дивизора $D$, т. е. коэффициент, с которым $E_{\sigma}$ входит в дивизор нулей функции $f_{i} \circ \pi$. Пусть $\underline{m}^{\sigma}:=\left(m_{1}^{\sigma}, \ldots, m_{r}^{\sigma}\right)$. 
Числа $m_{i}^{\sigma}$ могут быть описаны следующим образом. Пусть $\left(E_{\sigma} \circ E_{\delta}\right)$, $\sigma, \delta \in \Gamma,-$ матрица пересечений компонент $E_{\sigma}$ на многообразии разрешения $X$. Диагональные элементы $E_{\sigma} \circ E_{\sigma}$ этой матрицы - отрицательные целые числа. Внедиагональный элемент $E_{\sigma} \circ E_{\delta}(\sigma \neq \delta)$ равен единице, если компоненты $E_{\sigma}$ и $E_{\delta}$ пересекаются (в точке), и равен нулю в противном случае. Определитель матрицы $\left(E_{\sigma} \circ E_{\delta}\right)$ равен \pm 1 . (Точнее, он равен $(-1)^{\# \Gamma}$, где $\# \Gamma-$ количество компонент исключительного дивизора $D$.) Пусть $\left(m_{\sigma \delta}\right)=-\left(E_{\sigma} \circ E_{\delta}\right)^{-1}-$ минус матрица, обратная к матрице пересечений. Элементы $m_{\sigma \delta}$ этой матрицы положительные целые числа. Для $i=1, \ldots, r$ пусть собственный прообраз $\widetilde{C}_{i}$ компоненты $C_{i}$ кривой $C$ пересекает исключительный дивизор $D$ в точке компоненты $E_{\sigma_{i}}$. Тогда $m_{i}^{\sigma}=m_{\sigma \sigma_{i}}$.

Другой способ вычисления чисел $m_{i}^{\sigma}$ - рекурсия по процессу разрешения кривой $C$. В процессе разрешения кривой $C$ неприводимая компонента $E_{\sigma}$ исключительного дивизора $D$ появляется в результате раздутия некоторой точки $x$ исключительного дивизора на предыдущем шаге. Точка $x$ принадлежит одной или двум компонентам исключительного дивизора, а также может принадлежать собственным прообразам некоторых из неприводимых компонент кривой $C$. Если точка $x$ не принадлежит собственному прообразу $\widetilde{C}_{i}$ компоненты $C_{i}$ кривой $C$, то $m_{i}^{\sigma}$ равно сумме соответствующих чисел $m_{i}^{\delta}$ (одного или двух) для компонент $E_{\delta}$, проходящих через точку $x$. Если точка $x$ принадлежит собственному прообразу $\widetilde{C}_{i}$ компоненты $C_{i}$, то $m_{i}^{\sigma}$ равно указанной сумме плюс порядок ростка кривой $\widetilde{C}_{i}$ (собственного прообраза компоненты $C_{i}$ ) в точке $x$.

Обозначим через $\stackrel{\circ}{E}_{\sigma}$ “неособую часть" компоненты $E_{\sigma}$, т. е. саму компоненту $E_{\sigma}$ минус точки пересечения со всеми другими компонентами полного прообраза $\pi^{-1}(C)$ кривой $C$.

Теорема 6 [28], [24]. В терминах разрешения особенности кривой $C$ ряд Пуанкаре фильтрачии, соответствующей кривой, дается формулой

$$
P_{\left\{v_{i}\right\}}(\underline{t})=\prod_{\sigma \in \Gamma}\left(1-\underline{t}^{\frac{m^{\sigma}}{}}\right)^{-\chi\left(\stackrel{\circ}{E}_{\sigma}\right)} .
$$

ДокАЗАтельство. Докажем формулу (14) с точностью до членов степени не меньше $\underline{V}$, где $\underline{V}$ - любая наперед заданная точка решетки $\mathbb{Z}_{\geqslant 0}^{r}$. Сделав, если необходимо, дополнительные $\sigma$-процессы в точках пересечения компонент полного прообраза $\pi^{-1}(C)$ кривой $C$, мы можем предполагать, что для любого pостка $g \in \mathscr{O}_{\mathbb{C}^{2}, 0}$ такого, что $\underline{v}(g)<\underline{V}$, собственный прообраз кривой $\{g=0\}$ пересекает исключительный дивизор $D=\pi^{-1}(0)$ разрешения только в неособых точках, т. е. в точках

$$
\stackrel{\circ}{D}=\bigcup_{\sigma \in \Gamma} \stackrel{\circ}{E}_{\sigma}
$$

При таком дополнительном $\sigma$-процессе к исключительному дивизору добавляется компонента, неособая часть которой изоморфна проколотой аффинной прямой $\mathbb{C}^{*}=\mathbb{C} \backslash\{0\}$ и поэтому имеет нулевую эйлерову характеристику. Следовательно, при таком $\sigma$-процессе правая часть формулы (14) не меняется. 
Обозначим через $\mathscr{O}_{\mathbb{C}^{2}, 0}$ множество ростков $g \in \mathscr{O}_{\mathbb{C}^{2}, 0}$ с $\underline{v}(g)<\underline{V}$. Пусть $S^{k} Z=Z^{k} / S_{k}-k$-я симметрическая степень пространства $Z$. Обозначим через $Y$ конфигурационное пространство

$$
\prod_{\sigma \in \Gamma}\left(\bigsqcup_{k=0}^{\infty} S^{k} \stackrel{\circ}{E_{\sigma}}\right)=\bigsqcup_{\left\{k_{\sigma}\right\}}\left(\prod_{\sigma \in \Gamma} S^{k_{\sigma}} \stackrel{\circ}{E_{\sigma}}\right)
$$

эффективных дивизоров на неособой части $\stackrel{\circ}{D}=\bigcup_{\sigma \in \Gamma} \stackrel{\circ}{E_{\sigma}}$ исключительного дивизора $D$. Пусть $\underline{v}: Y \rightarrow \mathbb{Z}_{\geqslant 0}^{r}-$ функция со значениями в решетке $\mathbb{Z}_{\geqslant 0}^{r}$, переводящая все точки компоненты $\prod_{\sigma \in \Gamma} S^{k_{\sigma}} \stackrel{\circ}{E}_{\sigma}$ в $\sum_{\sigma \in \Gamma} k_{\sigma} \underline{m}^{\sigma} \in \mathbb{Z}_{\geqslant 0}^{r}$. Определим отображение $I: \mathbb{P} \mathscr{O}_{\mathbb{C}^{2}, 0}^{\frac{V}{4}} \rightarrow Y$ условием, что росток $g \in O_{\mathbb{C}^{2}, 0}^{V}$ (или, точнее, его класс в проективизации $\left.\mathbb{P} \mathscr{O} \frac{V}{\mathbb{C}^{2}}, 0\right)$ переходит в пересечение исключительного дивизора $D$ с собственным прообразом кривой $\{g=0\}$, т. е. в набор их точек пересечения с учетом кратностей. Нетрудно видеть, что $\underline{v} \circ I=\underline{v}$, т. е. отображение $I$ сохраняет функцию $\underline{v}$. Из формулы Фубини вытекает, что с точностью до указанных членов имеют место равенства

$$
P_{\left\{v_{i}\right\}}(\underline{t})=\int_{\mathbb{P} \mathscr{O} \frac{V}{\mathbb{C}^{2}, 0}} \underline{t}^{\underline{v}(g)} d \chi=\int_{Y} \chi\left(I^{-1}(y)\right) \underline{t}^{\underline{v}(y)} d \chi
$$

Лемма 1. Для любой точки $y \in Y$ прообраз $I^{-1}(y)-($ непустое комплексное) афбинное пространство.

ДокАЗАТЕЛЬСтво. Для $\sigma \in \Gamma, g \in O_{\mathbb{C}^{2}, 0}$ обозначим через $w_{\sigma}(g)$ порядок нуля поднятия $\tilde{g}=g \circ \pi$ функции $g$ на пространство $X$ разрешения вдоль компоненты $E_{\sigma}$ исключительного дивизора $D$. Утверждение следует из того, что образы $I(g)$ и $I\left(g^{\prime}\right)$ ростков $g$ и $g^{\prime}$ из $\mathscr{O} \frac{V}{\mathbb{C}^{2}, 0}$ равны тогда и только тогда, когда $g^{\prime}=\alpha g+h$, где $\alpha \in \mathbb{C}^{*}$, и $w_{\sigma}(h)>w_{\sigma}(g)\left(=w_{\sigma}\left(g^{\prime}\right)\right)$ для всех $\sigma \in \Gamma$.

Рассмотрим отношение $\Psi=\tilde{g}^{\prime} / \tilde{g}$ (здесь $\tilde{g}^{\prime}=g^{\prime} \circ \pi-$ поднятие функции $g^{\prime}$ на пространство разрешения). Это - мероморфная функция на пространстве $X$ разрешения. Если $g^{\prime}=\alpha g+h$, где $\alpha \in \mathbb{C}^{*}$, и $w_{\sigma}(h)>w_{\sigma}(g)\left(=w_{\sigma}\left(g^{\prime}\right)\right)$ для всех $\sigma \in \Gamma$, то $\Psi_{\mid D}=\alpha$. Следовательно, нули и полюсы функции $\Psi$ (т.е. нули числителя и знаменателя соответственно) поглощают друг друга на исключительном дивизоре $D$ и поэтому $I(g)=I\left(g^{\prime}\right)$. В обратную сторону, если $I(g)=I\left(g^{\prime}\right)$, то нули и полюсы функции $\Psi$ поглощают друг друга на исключительном дивизоре $D$. Следовательно, $\Psi$ - регулярная функция на исключительном дивизоре $D$ и поэтому постоянна (равна константе $\alpha$ ) на нем. Тогда $w_{\sigma}\left(g^{\prime}-\alpha g\right)>w_{\sigma}(g)=w_{\sigma}\left(g^{\prime}\right)$. Лемма 1 доказана.

Эйлерова характеристика комплексного аффинного пространства равна 1. Поэтому с точностью до указанных членов имеем

$$
P_{\left\{v_{i}\right\}}(\underline{t})=\int_{Y} \underline{t}^{\underline{v}(y)} d \chi=\prod_{\sigma \in \Gamma}\left(\sum_{k=0}^{\infty} \chi\left(S^{k} \stackrel{\circ}{E}_{\sigma}\right) \underline{t}^{k \underline{m}^{\sigma}}\right) .
$$


Воспользуемся следующей очень полезной формулой. (Она является частным случаем формулы Макдональда [29] для производящего ряда рангов групп гомологий симметрических степеней пространства.)

Лемма 2. Пусть $Z$ - оббединение клеток в конечном $C W$-комплексе. Тогда

$$
\sum_{k=0}^{\infty} \chi\left(S^{k} Z\right) t^{k}=(1-t)^{-\chi(Z)}
$$

ДокАЗАтЕЛьСтво. Если $Z=Z_{1} \cup Z_{2}, Z_{1} \cap Z_{2}=\varnothing$, то симметрическая степень $S^{k} Z$ является несвязным объединением произведений $S^{i} Z_{1} \times S^{k-i} Z_{2}$ для $i=0, \ldots, k$. Поэтому

$$
\sum_{k=0}^{\infty} \chi\left(S^{k} Z\right) t^{k}=\sum_{k=0}^{\infty} \chi\left(S^{k} Z_{1}\right) t^{k} \cdot \sum_{k=0}^{\infty} \chi\left(S^{k} Z_{2}\right) t^{k}
$$

Поскольку $(1-t)^{-\chi(Z)}=(1-t)^{-\chi\left(Z_{1}\right)}(1-t)^{-\chi\left(Z_{2}\right)}$, утверждение достаточно доказать для одной клетки. Это может быть сделано, например, индукцией по размерности клетки. Очевидно, что утверждение верно для нульмерной клетки (т. е. для точки) и поэтому верно для (гомотопически ей эквивалентного) замкнутого (!) шара любой размерности. Замкнутый шар размерности $n$ представим в виде $C W$-комплекса с одной нульмерной клеткой, одной $(n-1)$-мерной и одной $n$-мерной. Из справедливости утверждения для шара и для клеток размерностей меньше $n$ и из описанной мультипликативности левой и правой частей равенства (15) вытекает его справедливость для клетки размерности $n$. Лемма 2 доказана.

В силу (15) имеем

$$
P_{\left\{v_{i}\right\}}(\underline{t})=\prod_{\sigma \in \Gamma}\left(\sum_{k=0}^{\infty} \chi\left(S^{k} \stackrel{\circ}{E}_{\sigma}\right) \underline{t}^{k \underline{m}^{\sigma}}\right)=\prod_{\sigma \in \Gamma}\left(1-\underline{t}^{\underline{m}^{\sigma}}\right)^{-\chi\left(\stackrel{\circ}{E}_{\sigma}\right)} .
$$

Теорема 6 доказана.

Пример. Пусть $C=\left\{y\left(y^{2}-x^{3}\right)=0\right\}, C_{1}=\{y=0\}, C_{2}=\left\{y^{2}-x^{3}=0\right\}$. Граф (минимального) разрешения кривой $C$ изображен на рис. 1 . Здесь компоненты исключительного дивизора изображены отрезками, компоненты собственного прообраза кривой $C$ - стрелками. Около каждой компоненты $E_{\sigma}$ исключительного дивизора написан элемент $\underline{m}^{\sigma}=\left(m_{1}^{\sigma}, m_{2}^{\sigma}\right)$. Неособая часть $\stackrel{\circ}{E}_{i}$ компоненты $E_{i}(i=1,2,3)$ является (комплексной) проективной прямой без одной, двух и трех точек соответственно. Поэтому $\chi\left(\stackrel{\circ}{E}_{1}\right)=1, \chi\left(\stackrel{\circ}{E}_{2}\right)=0$, $\chi\left(\stackrel{\circ}{E}_{3}\right)=-1$. Формула $(14)$ дает

$$
P_{\left\{v_{1}, v_{2}\right\}}\left(t_{1}, t_{2}\right)=\frac{1-t_{1}^{3} t_{2}^{6}}{1-t_{1} t_{2}^{2}}
$$

Выражение в правой части формулы (14) напоминает выражение в формуле А'Кампо для дзета-функции монодромии ростка $f$ : оно совпадает с дзета- 


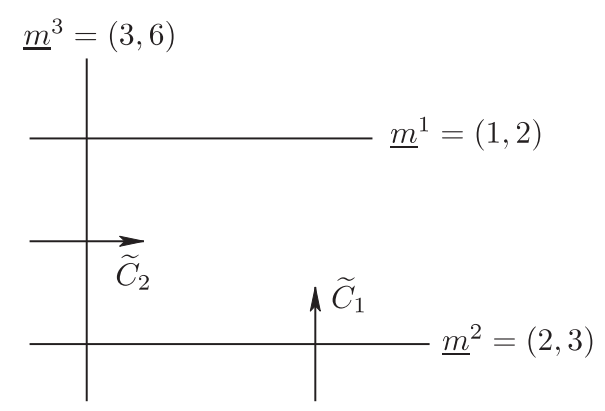

Рис. 1. Граф разрешения кривой $C$

функцией монодромии при $r=1$, а в общем случае превращается в нее при подстановке $t_{1}=\cdots=t_{r}=t$. Как следует из [30], при $r>1$ это выражение совпадает с многочленом Александера $\Delta_{C}\left(t_{1}, \ldots, t_{r}\right)$ (от нескольких переменных) $r$-компонентного зацепления $C \cap S_{\varepsilon}^{3} \subset S_{\varepsilon}^{3}$, где $S_{\varepsilon}^{3}$ - сфера малого радиуса $\varepsilon$ с центром в начале координат в $\mathbb{C}^{2}$. При $r=1$ дзета-функция монодромии ростка $f$ равна многочлену Александера указанного зацепления (в этом случае узла), деленному на $1-t$.

ЗАмечАния. 1. Многочлен Александера зацепления определен, вообе говоря, только с точностью до умножения на \pm моном. Здесь многочлен Александера предполагается нормированным таким образом, что он действительно является многочленом (а не многочленом Лорана), а его свободный член (значение в нуле) равно 1. В рассматриваемом случае это возможно.

2. В действительности, равенство правой части формулы (14) при $r>1$ многочлену Александера соответствующего зацепления легко следует из формулы А'Кампо, если заметить, что дзета-функция монодромии ростка $\prod_{i=1}^{r} f_{i}^{s_{i}}$
$\left(s_{i} \in \mathbb{Z}_{>0}\right)$ совпадает с $\Delta_{C}\left(t^{s_{1}}, \ldots, t^{s_{r}}\right)$.

СледствиЕ [31], [32], [28]. Ряд Пуанкаре (одноиндексной) филътрации, соответствующей неприводимой кривой $C$, совпадает с многочленом Александера алгебраического узла, соответствующего особенности кривой $C$, деленнъмм на 1 - t. При $r>1$ ряд Пуанкаре мультииндексной фильтрации, соответствующей кривой $C=\bigcup_{i=1}^{r} C_{i}$, совпадает с многочленом Александера алгебраического зацепления, соответствующего особенности кривой $C$.

ЗАмечАниЕ. Указанное совпадение не имеет убедительного объяснения, отличного от явного вычисления обеих его частей (ряда Пуанкаре и многочлена Александера) в общих терминах, например, в терминах разрешения особенности.

Аналоги указанного совпадения имеются для алгебраических кривых на комплексной плоскости с одним местом на бесконечности: [33], для кривых на рациональной двойной особенности поверхности типа $E_{8}$ (ее зацепление является гомологической 3-сферой): [34]. Для бо́льших размерностей аналоги 
приведенных выше рассуждений показывают, что дзета-функция монодромии равна интегралу по отношению к эйлеровой характеристике по исключительному дивизору, а ряд Пуанкаре фильтрации - интегралу по конфигурационному пространству (эффективных) дивизоров на нем (или, точнее, по его подпространству, состоящему из пересечений с дивизорами Картье). Поэтому можно ожидать не совпадения дзета-функции монодромии с рядом Пуанкаре, а их связи с участием некоторой двойственности. Так, для квазиоднородных особенностей описана подобная связь, в которой участвует так называемая двойственность К. Саито: [35]-[37].

Имеются аналоги теоремы 6 для фильтраций, определенных наборами дивизориальных нормирований на кольце $\mathscr{O}_{\mathbb{C}^{2}, 0}[38]$ и на кольце ростков функций на рациональной особенности поверхности [39], для эквивариантного (в смысле [40]) ряда Пуанкаре дивизориальной фильтрации на универсальном абелевом накрытии рациональной особенности поверхности [41]. А. Немети обнаружил, что вычисление эквивариантного ряда Пуанкаре дивизориальной фильтрации на кольце функций на универсальном абелевом накрытии особенности поверхности тесно связано с вычислением инвариантов Зайберга-Виттена соответствующего зацепления: [42], [43].

\section{7. Универсальная эйлерова характеристика и мотивная мера на пространстве функций}

Эйлерова характеристика является не единственным аддитивным инвариантом на алгебре (комплексных) конструктивных множеств. Другим примером такого инварианта (со значениями в кольце $\mathbb{Z}[u, v]$ многочленов от двух переменных) является многочлен Ходжа-Делиня: [44]-[46]. Многочлен ХоджаДелиня $e_{X}(u, v)$ квазипроективного пространства $X$ может быть определен следующими двумя свойствами:

1) для проективного (неособого) многообразия $X$ многочлен $e_{X}(u, v)$ равен $\sum h_{X}^{i j}(-u)^{i}(-v)^{j}$, где $h_{X}^{i j}$ - числа Ходжа многообразия $X$;

2) если $Y$ - замкнутое по Зарискому подмножество пространства $X$, то $e_{X}(u, v)=e_{Y}(u, v)+e_{X \backslash Y}(u, v)$.

Из этих свойств единственность многочлена Ходжа-Делиня легко следует, в то время как его существование является нетривиальным фактом.

В свою очередь, и эйлерова характеристика и многочлен Ходжа-Делиня являются специализациями универсального аддитивного инварианта, определяемого очевидным образом.

ОПРЕДЕЛЕНИЕ. Кольцо Гротендика $K_{0}\left(\mathscr{V}_{\mathbb{C}}\right)$ комплексных квазипроективнъх пространств - это абелева группа, порожденная классами $[X]$ всех квазипроективных пространств $X$, по модулю следующих соотношений:

1) если пространства $X$ и $Y$ изоморфны, то $[X]=[Y]$;

2) если $Y$ - замкнутое по Зарискому подпространство пространства $X$, то $[X]=[Y]+[X \backslash Y]$. 
Умножение в $K_{0}\left(\mathscr{V}_{\mathbb{C}}\right)$ определяется декартовым произведением пространств. Единицей в кольце $K_{0}\left(\mathscr{V}_{\mathbb{C}}\right)$ является класс пространства, состоящего из одной точки.

ЗАмЕчАниЕ. Здесь существенно, что квазипроективные пространства рассматриваются именно как множества, а не как схемы, т. е. без соответствующих структурных пучков. Иначе соотношение 2) теряет смысл.

Если $p: E \rightarrow B$ - (алгебраическое) отображение квазипроективных пространств, являющееся локально тривиальным расслоением со слоем $F$ в обычной топологии, то, вообще говоря, $[E] \neq[B][F]$. Например, отображение $\mathbb{C}^{*} \rightarrow \mathbb{C}^{*}$ $\left(\mathbb{C}^{*}=\mathbb{C} \backslash\{0\}\right)$, переводящее $z$ в $z^{2}$, является локально тривиальным расслоением (накрытием), слоем которого является пара точек. Однако $\left[\mathbb{C}^{*}\right] \neq 2\left[\mathbb{C}^{*}\right]$. Равенство $[E]=[B][F]$ выполняется для расслоения $p: E \rightarrow B$, локально тривиального в топологии Зариского.

Класс $\left[\mathbb{A}_{\mathbb{C}}^{1}\right] \in K_{0}\left(\mathscr{V}_{\mathbb{C}}\right)$ комплексной аффинной прямой $\mathbb{A}_{\mathbb{C}}^{1}$ играет особую роль и обозначается через $\mathbb{L}$. Кольцо Гротендика $K_{0}\left(\mathscr{V}_{\mathbb{C}}\right)$ трудно обозримо. Например, неизвестно, является ли $\mathbb{L}$ делителем нуля в нем. (При этом естественное отображение $\mathbb{Z}[\mathbb{L}] \rightarrow K_{0}\left(\mathscr{V}_{\mathbb{C}}\right)$ является вложением.) Эйлерова характеристика и многочлен Ходжа-Делиня являются гомоморфизмами из кольца Гротендика $K_{0}\left(\mathscr{V}_{\mathbb{C}}\right)$ в кольцо $\mathbb{Z}$ целых чисел и в кольцо $\mathbb{Z}[u, v]$ многочленов от двух переменных соответственно. Они обычно и используются в приложениях. В ряде случаев (например, для определения мотивного интегрирования: см. ниже) разумно (или, скорее, необходимо) рассматривать локализацию $K_{0}\left(\mathscr{V}_{\mathbb{C}}\right)\left[\mathbb{L}^{-1}\right]$ кольца Гротендика $K_{0}\left(\mathscr{V}_{\mathbb{C}}\right)$ по классу $\mathbb{L}$ и ее пополнение.

$\Phi$. Биттнер [47] показала, что кольцо $K_{0}\left(\mathscr{V}_{\mathbb{C}}\right)$ может быть определено как свободная абелева группа, порожденная классами изоморфизма $[X]$ гладких проективных многообразий, по модулю соотношения $[X]-[Y]=\left[\mathrm{Bl}_{Y} X\right]-[E]$, где $Y \subset X$ - гладкое замкнутое подмногообразие, $\mathrm{Bl}_{Y} X$ - раздутие $X$ вдоль $Y$, a $E$ - исключительный дивизор этого раздутия (проективизация нормального расслоения). Это выводится из так называемой слабой теоремы факторизации Я. Влодарчика: [48], [49].

$\mathrm{Kласс}[X] \in K_{0}\left(\mathscr{V}_{\mathbb{C}}\right)$ является универсальным аддитивным инвариантом квазипроективных пространств в том смысле, что любой аддитивный инвариант является гомоморфизмом кольца Гротендика $K_{0}\left(\mathscr{V}_{\mathbb{C}}\right)$, рассматриваемого как абелева группа, в группу значений инварианта. Если инвариант мультипликативен (в обычном смысле, т. е. для декартовых произведений пространств), то он является кольцевым гомоморфизмом кольца Гротендика $K_{0}\left(\mathscr{V}_{\mathbb{C}}\right)$ в кольцо значений инварианта. Так, эйлерова характеристика и многочлен ХоджаДелиня являются кольцевыми гомоморфизмами в кольца $\mathbb{Z}$ целых чисел и $\mathbb{Z}[u, v]$ многочленов от двух переменных соответственно.

Будучи универсальным аддитивным инвариантом, класс $[X] \in K_{0}\left(\mathscr{V}_{\mathbb{C}}\right)$ может рассматриваться как обобщенная (универсальная) эйлерова характеристика квазипроективного пространства $X$. Для удобства обозначений (в частности, соответствующего интеграла по отношению к эйлеровой характеристике) мы будем использовать для $[X]$ обозначение $\chi_{g}(X)$. 
ЗАмЕчАниЕ. Можно также рассматривать понятие полукольца Гротендика $S_{0}\left(\mathscr{V}_{\mathbb{C}}\right)$ комплексных квазипроективных пространств, заменяя выше слово "группа" словом "полугруппа". Элементы полукольца $S_{0}\left(\mathscr{V}_{\mathbb{C}}\right)$ имеют несколько больше геометрического смысла: они представляются "настоящими" квазипроективными пространствами (не виртуальными).

Если $X$ - конструктивное (конечномерное) множество, а $\psi: X \rightarrow G-$ конструктивная функция на нем со значениями в абелевой группе $G$, то интеграл функции $\psi$ по пространству $X$ можно рассматривать по отношению к обобщенной эйлеровой характеристике:

$$
\int_{X} \psi d \chi_{g}=\sum_{a \in G} \chi_{g}\left(\psi^{-1}(a)\right) a=\sum_{a \in G}\left[\psi^{-1}(a)\right] a \in K_{0}\left(\mathscr{V}_{\mathbb{C}}\right) \otimes \mathbb{Z}[G],
$$

где $\mathbb{Z}[G]$ - групповое кольцо группы $G$.

Для интегрирования по бесконечномерным пространствам кольцо значений меры (кольцо Гротендика квазипроективных многообразий) $K_{0}\left(\mathscr{V}_{\mathbb{C}}\right)$ должно быть несколько модифицировано. Пусть $K_{0}\left(\mathscr{V}_{\mathbb{C}}\right)_{(\mathbb{L})}\left(=K_{0}\left(\mathscr{V}_{\mathbb{C}}\right)\left[\mathbb{L}^{-1}\right]\right)-$ локализация кольца $K_{0}\left(\mathscr{V}_{\mathbb{C}}\right)$ по классу $\mathbb{L}$ комплексной аффинной прямой (т. е. по порожденному им идеалу). Если $X$ - цилиндрическое подмножество в проективизации $\mathbb{P} \mathscr{O}_{V, 0}, X=\pi_{k}^{-1}(Y), Y \subset \mathbb{P} J_{V, 0}^{k}$, то его обобщенную эйлерову характеристику нельзя определить формулой $\chi_{g}(X)=[Y]$ : результат будет зависеть от $k$. Вместо этого ее следует определить как элемент кольца $K_{0}\left(\mathscr{V}_{\mathbb{C}}\right)_{(\mathbb{L})}$ (локализации кольца Гротендика $\left.K_{0}\left(\mathscr{V}_{\mathbb{C}}\right)\right)$ формулой $\chi_{g}(X)=\mathbb{L}^{-d_{k}}[Y]$, где $d_{k}=\operatorname{dim} J_{V, 0}^{k}$. Действительно, если также $X=\pi_{k^{\prime}}^{-1}\left(Y^{\prime}\right), Y^{\prime} \subset \mathbb{P} J_{V, 0}^{k^{\prime}}, k^{\prime} \geqslant k$, то $Y^{\prime}$ является локально тривиальным (в топологии Зариского) расслоением над $Y$, слоем которого является комплексное аффинное пространство размерности $d_{k^{\prime}}-d_{k}$. Поэтому $\left[Y^{\prime}\right]=\mathbb{L}^{d_{k^{\prime}}-d_{k}}[Y]$.

Так определенная обобщенная эйлерова характеристика является аддитивной функцией на алгебре цилиндрических подмножеств проективизации $\mathbb{P} \mathscr{O}_{V, 0}$ и поэтому может использоваться в качестве меры для соответствующего определения интеграла. Функция $\psi: \mathbb{P} \mathscr{O}_{V, 0} \rightarrow G$ (со значениями в абелевой группе $G$ ) называется цилиндрической, если для любого ненулевого $a \in G$ множество уровня $\psi^{-1}(a)$ цилиндрично. Интеграл (цилиндрической) функции $\psi$ по отношению к обобщенной эйлеровой характеристике определяется формулой

$$
\int_{\mathbb{P} \mathscr{O}_{V, 0}} \psi d \chi_{g}=\sum_{a \in G, a \neq 0} \chi_{g}\left(\psi^{-1}(a)\right) a \in K_{0}\left(\mathscr{V}_{\mathbb{C}}\right)_{(\mathbb{L})} \otimes \mathbb{Z}[G],
$$

если соответствующая интегральная сумма имеет смысл в указанной группе. (В противном случае функция $\psi$ не является интегрируемой.)

В правой части формулы-определения ряда Пуанкаре мультииндексной фильтрации

$$
P_{\left\{v_{i}\right\}}\left(t_{1}, \ldots, t_{r}\right)=\int_{\mathbb{P} \mathscr{O}_{V, 0}} \underline{t}^{\underline{v}(g)} d \chi
$$

можно заменить интегрирование по эйлеровой характеристике интегрированием по обобщенной эйлеровой характеристике со значениями в локализации $K_{0}\left(\mathscr{V}_{\mathbb{C}}\right)_{(\mathbb{L})}$ кольца Гротендика квазипроективных многообразий $K_{0}\left(\mathscr{V}_{\mathbb{C}}\right)$. 
Такая замена приводит к определению обобщенного ряда Пуанкаре фильтрации [50]:

$$
P_{\left\{v_{i}\right\}}^{\text {gen }}\left(t_{1}, \ldots, t_{r}\right)=\int_{\mathbb{P} \mathscr{O}_{V, 0}} \underline{t}^{\underline{v}(g)} d \chi_{g} .
$$

С формальной точки зрения результат интегрирования принадлежит кольцу $K_{0}\left(\mathscr{V}_{\mathbb{C}}\right)_{(\mathbb{L})}\left[\left[t_{1}, \ldots, t_{r}\right]\right]$ степенных рядов от переменных $t_{1}, \ldots, t_{r}$ с коэффициентами из кольца $K_{0}\left(\mathscr{V}_{\mathbb{C}}\right)_{(\mathbb{L})}$. Однако нетрудно заметить, что все коэффициенты обобщенного ряда Пуанкаре являются многочленами от $q=\mathbb{L}^{-1}$. Поэтому обобщенный ряд Пуанкаре фильтрации является элементом кольца $\mathbb{Z}[q]\left[\left[t_{1}, \ldots, t_{r}\right]\right]$ и может записываться в виде $P_{\left\{v_{i}\right\}}^{\text {gen }}\left(t_{1}, \ldots, t_{r} ; q\right)$. Он может рассматриваться как деформация (по параметру $q$ ) обычного ряда Пуанкаре. Подстановка $q=1$ превращает обобщенный ряд Пуанкаре в обычный.

Как показано выше, ряд Пуанкаре мультииндексной фильтрации, определенной особенностью плоской кривой, совпадает с многочленом Александера соответствующего (алгебраического) зацепления. Одной из деформаций многочлена Александера является производящий ряд гомологий Хегора-Флоера, определенных П. Ожватом и 3. Сабо: см., например, [51], [52]. Е. А. Горский [53] показал, что для неприводимых особенностей плоских кривых производящий ряд гомологий Хегора-Флоера соответствующего узла с точностью до простой замены совпадает с обобщенным рядом Пуанкаре соответствующей фильтрации.

\section{8. Мотивное интегрирование}

Мотивное интегрирование - это интегрирование по отношению к так называемой мотивной мере по пространству дуг на многообразии. Оно было введено М. Концевичем [2] как метод доказательства совпадения чисел Ходжа так называемых крепантных разрешений пространств Калаби-Яу. Подробное описание этого понятия можно найти в [54], [55].

Мотивная мера принимает свои значения в некоторой модификации (пополнении локализации) кольца Гротендика $K_{0}\left(\mathscr{V}_{\mathbb{C}}\right)$ квазипроективных многообразий. Пусть, как и выше, $K_{0}\left(\mathscr{V}_{\mathbb{C}}\right)_{(\mathbb{L})}$ - локализация кольца Гротендика $K_{0}\left(\mathscr{V}_{\mathbb{C}}\right)$ квазипроективных многообразий по классу $\mathbb{L}$ комплексной аффинной прямой. Рассмотрим фильтрацию кольца $K_{0}\left(\mathscr{V}_{\mathbb{C}}\right)_{(\mathbb{L})}$ подгруппами $F_{i}, i \in \mathbb{Z}$, порожденными элементами вида $[V] \mathbb{L}^{-j}$, где $V$ - квазипроективное пространство размерности $\leqslant j-i$. Пусть $\mathscr{M}-$ пополнение кольца $K_{0}\left(\mathscr{V}_{\mathbb{C}}\right)_{(\mathbb{L})}$ по этой фильтрации:

$$
\mathscr{M}=\lim _{i \rightarrow \infty} K_{0}\left(\mathscr{V}_{\mathbb{C}}\right)_{(\mathbb{L})} / F_{i}
$$

Элементы кольца $\mathscr{M}$ представимы в виде $\sum_{i=1}^{\infty}\left[X_{i}\right] \mathbb{L}^{-r_{i}}$, где $\operatorname{dim} X_{i}-r_{i} \rightarrow-\infty$ при $i \rightarrow \infty$. При этом неясно, может ли нетривиальный ряд такого вида представлять нулевой элемент кольца $\mathscr{M}$. Это связано с тем, что неизвестно, равно ли нулю пересечение $\bigcap_{i} F_{i}$ элементов фильтрации $\left\{F_{i}\right\}$. (Это пересечение совпадает с ядром естественного гомоморфизма $K_{0}\left(\mathscr{V}_{\mathbb{C}}\right)_{(\mathbb{L})} \rightarrow \mathscr{M}$.) 
Нетрудно видеть, что эйлерова характеристика не продолжается с $K_{0}\left(\mathscr{V}_{\mathbb{C}}\right)_{(\mathbb{L})}$ на $\mathscr{M}$. В то же время многочлен Ходжа-Делиня продолжается до гомоморфизма кольца $\mathscr{M}$ в кольцо, являющееся пополнением кольца $\mathbb{Z}[u, v]_{(u v)}$ (локализации кольца многочленов от двух переменных по элементу $(u v))$ по фильтрации подгруппами $L_{i}, i \in \mathbb{Z}$, порожденными элементами вида $P(u, v)(u v)^{-j}$, где $P \in \mathbb{Z}[u, v], \operatorname{deg} P \leqslant j-i$.

Идея определения мотивной меры на пространстве дуг в некоторой степени аналогична описанной выше конструкции меры на пространстве ростков функций на многообразии (или на его проективизации) со значениями в локализации $K_{0}\left(\mathscr{V}_{\mathbb{C}}\right)_{(\mathbb{L})}$ кольца Гротендика $K_{0}\left(\mathscr{V}_{\mathbb{C}}\right)$.

Пусть $X-n$-мерное квазипроективное многообразие, $J^{k}(X)$ - пространство $k$-струй дуг на $X$, т. е. струй ростков (комплексно аналитических или формальных) отображений $(\mathbb{C}, 0) \rightarrow X$. Пространство $k$-струй дуг $J^{k}(X)$ является квазипроективным многообразием. Оно расслоено над $X$. Слоем над точкой $x \in X$ является пространство $k$-струй дуг в точке $x$, изоморфное (комплексному) аффинному пространству размерности $k n$. Формальной дугой на многообразии $X$ в точке $x$ называется формальное отображение $(\mathbb{C}, 0) \rightarrow(X, x)$ (т. е. в локальных координатах на $X$ с началом в точке $x$, набор формальных степенных рядов $\left(x_{1}(t), \ldots, x_{n}(t)\right)$ с $\left.x_{i}(0)=0\right)$. Пусть $\mathscr{L}(X)-$ пространство всех формальных дуг на $X$ (во всех точках). Пространство $\mathscr{L}(X)$ расслоено над $X$. Слоем над точкой $x \in X$ является пространство формальных дуг в точке $x$. Имеются естественные отображения

$$
\pi_{k}: \mathscr{L}(X) \rightarrow J^{k}(X), \quad \pi_{k, \ell}: J^{k}(X) \rightarrow J^{\ell}(X), \quad k \geqslant \ell .
$$

ОПРЕДЕЛЕНИЕ. Подмножество $C \subset \mathscr{L}(X)$ пространства формальных дуг на многообразии $X$ называется иилиндрическим, если $C=\pi_{k}^{-1}(B)$ для некоторого $k$ и для конструктивного подмножества $B \subset J^{k}(X)$.

Множество цилиндрических подмножеств пространства $\mathscr{L}(X)$ является алгеброй множеств. Размерностью (относительной) $\operatorname{dim} C$ цилиндрического подмножества $C=\pi_{k}^{-1}(B), B \subset J^{k}(X)$, называется $\operatorname{dim} B-k n(n=\operatorname{dim} X)$. Мера (мотивная) $\mu(C)$ цилиндрического подмножества $C=\pi_{k}^{-1}(B), B \subset J^{k}(X)$, определяется как $[B] \mathbb{L}^{-k n} \in K_{0}\left(\mathscr{V}_{\mathbb{C}}\right)_{(\mathbb{L})}$.

ОПРЕДЕЛЕНИЕ. Подмножество $C \subset \mathscr{L}(X)$ называется измеримым, если для любого $m$ существуют цилиндрическое подмножество $C_{m}$ и цилиндрические подмножества $D_{m, i}, i \in \mathbb{N}$, такие, что симметрическая разность $C \triangle C_{m}=$ $\left(C \backslash C_{m}\right) \cup\left(C_{m} \backslash C\right)$ подмножеств $C$ и $C_{m}$ содержится в (бесконечном) объединении $\bigcup_{i \in \mathbb{N}} D_{m, i}$ и $\operatorname{dim} D_{m, i} \leqslant-m$ для каждого $i$.

Множество измеримых подмножеств пространства $\mathscr{L}(X)$ является сигмаалгеброй множеств.

ОПРЕДЕЛЕНИЕ. Мотивная мера $\mu(C)$ измеримого подмножества $C \subset \mathscr{L}(X)-$ это $\lim _{m \rightarrow \infty} \mu\left(C_{m}\right) \in \mathscr{M}$, где $C_{m}, m \in \mathbb{N},-$ цилиндрические подмножества, описанные в предыдущем определении. 
Существование предела $\lim _{m \rightarrow \infty} \mu\left(C_{m}\right) \in \mathscr{M}$ вытекает из определения измеримого подмножества. Можно доказать (это не тривиально), что определение мотивной меры измеримого множества корректно, т. е. не зависит от выбора цилиндрических подмножеств $C_{m}$ и $D_{m, i}$. Измеримыми подмножествами являются, в частности, (бесконечные) несвязные объединения вида $\bigcup_{i=1}^{\infty} C_{i}$, где $C_{i}-$ цилиндрические подмножества такие, что предел $\mu\left(C_{i}\right)$ при $i \rightarrow \infty$ равен нулю в кольце $\mathscr{M}$ (и дополнения к таким множествам). Мотивная мера $\mu(C)$ такого $C=\bigcup_{i=1}^{\infty} C_{i}$ равна $\sum_{i=1}^{\infty} \mu\left(C_{i}\right)$. Мотивная мера является счетно аддитивной.

ОПРЕДЕЛЕНИЕ. Функция $\psi: \mathscr{L}(X) \rightarrow G$ со значениями в абелевой группе $G$ называется простой, если область интегрирования $\mathscr{L}(X)$ можно разбить на (не более чем) счетное число измеримых подмножеств $A_{j}$ так, что на каждом из них функция $\psi$ принимает постоянное значение, равное $\psi_{j}$. Для простой функции $\psi$ ее мотивный интеграл - это

$$
\int_{\mathscr{L}(X)} \psi d \mu=\sum_{j} \psi_{j} \mu\left(A_{j}\right)
$$

(при условии, что сумма в правой части имеет смысл в группе $\mathbb{Z}[G] \otimes \mathscr{M}$ ).

Нетрудно видеть, что для неособого квазипроективного многообразия $X$ имеет место равенство

$$
\int_{\mathscr{L}(X)} 1 d \mu=[X]
$$

Одним из эффективных инструментов в теории мотивного интегрирования является формула замены переменных в мотивном интеграле, предложенная Я. Денефом и Ф. Лозером.

Пусть $h: Y \rightarrow X$ - собственный бирациональный морфизм гладких алгебраических многообразий размерности $d$. Пусть $J=h^{*} K_{X}-K_{Y}$ - относительный канонический дивизор на $Y$ (локально - множество нулей якобиана отображения). Он определяет функцию $\operatorname{ord}_{J}$ на пространстве дуг на $Y$ - индекс пересечения дуги с дивизором. Тогда имеет место следующая формула замены переменных в мотивном интеграле.

Tеорема 7 (см. [4]). Пусть $A$ - измеримое подмножество в пространстве $\mathscr{L}(X)$ дуг на $X, \alpha-$ простая функиия на нем. Тогда

$$
\int_{A} \alpha d \mu=\int_{h^{-1}(A)}\left(h^{*} \alpha\right) \mathbb{L}^{-\operatorname{ord}_{J}} d \mu .
$$

В качестве примера применения формулы замены переменных в мотивном интеграле приведем доказательство принадлежащего В. Батыреву результата о равенстве чисел Ходжа двух бирационально эквивалентных многообразий Калаби-Яу.

ПРеДЛОЖЕНИЕ 3 [9]. Пусть $X$ и $Y$ - бирационально эквивалентные многообразия Калаби-Яу. Тогда $e_{X}(u, v)=e_{Y}(u, v)$. 
ДокАЗАтЕльство. Мы докажем формально более сильное утверждение, а именно совпадение классов $[X]$ и $[Y]$ в кольце Гротендика квазипроективных многообразий. По теореме Хиронаки существует многообразие $Z$ вместе с регулярными отображениями $p: Z \rightarrow X$ и $q: Z \rightarrow Y$, являющимися бирациональными морфизмами.

Имеют место следующие равенства:

$$
[X]=\int_{\mathscr{L}(X)} 1 d \mu, \quad[Y]=\int_{\mathscr{L}(Y)} 1 d \mu .
$$

Применяя к отображениям $p$ и $q$ формулу замены переменных в мотивном интеграле, получаем:

$$
\begin{aligned}
{[X] } & =\int_{\mathscr{L}(X)} 1 d \mu=\int_{\mathscr{L}(Z)} \mathbb{L}^{-\gamma \circ\left(K_{Z}-p^{*} K_{X}\right)} d \mu=\int_{\mathscr{L}(Z)} \mathbb{L}^{-\gamma \circ K_{Z}} d \mu \\
& =\int_{\mathscr{L}(Z)} \mathbb{L}^{-\gamma \circ\left(K_{Z}-p^{*} K_{Y}\right)} d \mu=\int_{\mathscr{L}(Y)} 1 d \mu=[Y] .
\end{aligned}
$$

Таким образом, классы $[X]$ и $[Y]$ в кольце Гротендика совпадают, и, следовательно, совпадают и их многочлены Ходжа-Делиня. Предложение 3 доказано.

В качестве еще одного примера применения мотивного интегрирования можно указать результаты М. Мустаца о выражении так называемых “лог-канонических порогов" через мотивный интеграл: [56], [57] и другие работы.

Аналог мотивной меры может быть определен и на пространстве непараметризованных дуг: [58].

\section{9. Степенная структура над кольцом Гротендика квазипроективных многообразий}

Как уже говорилось, рассмотрение вместо обычной эйлеровой характеристики (со значениями в кольце $\mathbb{Z}$ целых чисел) обобщенной (универсальной) эйлеровой характеристики со значениями в кольце Гротендика $K_{0}\left(\mathscr{V}_{\mathbb{C}}\right)$ квазипроективных многообразий позволяет в некоторых случаях строить мотивные аналоги "обычных" инвариантов, которые, как правило, являются более тонкими. Примеры таких мотивных аналогов инвариантов могут быть найдены, в частности, в [6]-[8], [59]. При этом может возникать проблема интерпретации выражений, определяющих "обычные" инварианты в терминах эйлеровой характеристики, в случае замены обычной эйлеровой характеристики на универсальную. Так, подобная модификация формулы А'Кампо

$$
\zeta_{f}(t)=\int_{\ldots}\left(1-t^{m}\right)^{-d \chi}
$$

где интеграл может браться либо по исключительному дивизору разрешения ростка $f$, либо по пространству $\mathscr{L}_{0}^{*} / \mathbb{C}^{*}$ дуг, профакторизованному по $\mathbb{C}^{*}$-действию, либо по пространству $\mathscr{B}_{0}$ ветвей (т. е. непараметризованных дуг; см. [58]), приводит к проблеме интерпретации выражения

$$
\left(1-t^{m}\right)^{-\chi_{g}(X)}=\left(1-t^{m}\right)^{-[X]} .
$$


Выражение $(1-t)^{-[X]}$ должно обладать свойством мультипликативности

$$
(1-t)^{-\left[X_{1} \sqcup X_{2}\right]}=(1-t)^{-\left[X_{1}\right]}(1-t)^{-\left[X_{2}\right]}
$$

и удовлетворять условию нормировки

$$
(1-t)^{-1}=1+t+t^{2}+\cdots
$$

Этими свойствами обладает дзета-функция Капранова пространства $X[60]$ :

$$
\zeta_{X}(t)=1+[X] t+\left[S^{2} X\right] t^{2}+\left[S^{3} X\right] t^{3}+\cdots,
$$

где $S^{k} X=X^{k} / S_{k}-k$-я симметрическая степень пространства $X$. (Это следует непосредственно из того, что $S^{k}\left(X_{1} \bigsqcup X_{2}\right)=\bigsqcup_{i=0}^{k} S^{i} X_{1} \times S^{k-i} X_{2}$.) Это дает возможность интерпретировать $\left(1-t^{m}\right)^{-[X]}$ как $\zeta_{X}\left(t^{m}\right)\left[\right.$ т. е. рассматривать $\zeta_{X}\left(t^{m}\right)$ как (мотивный) аналог выражения $\left.\left(1-t^{m}\right)^{-\chi(X)}\right]$.

Можно показать, что

$$
\zeta_{\mathbb{L}^{s}}(t)=\left(1-\mathbb{L}^{s} t\right)^{-1} .
$$

Известно, что дзета-функция Капранова $\zeta_{X}(t)$, вообще говоря, не является рациональной функцией [61]. Поэтому ее можно рассматривать только как формальный ряд

$$
\zeta_{X}(t) \in 1+t K_{0}\left(\mathscr{V}_{\mathbb{C}}\right)[[t]]
$$

ЗАмечАниЕ. Для кольца $R$ ряд $\lambda_{a}(t) \in 1+t R[[t]]$, определенный для всех $a \in$ $R$ так, что $\lambda_{a+b}(t)=\lambda_{a}(t) \lambda_{b}(t), \lambda_{a}(t)=1+a t+($ члены более высокой степени), называется пре- $\lambda$-структурой на кольце $R$ (см., например, [62]).

Таким образом дзета-функция Капранова определяет пре- $\lambda$-структуру на кольце Гротендика $K_{0}\left(\mathscr{V}_{\mathbb{C}}\right)$ квазипроективных многообразий.

Аналогичный подход к выражению из теоремы 4 приводит к задаче интерпретации выражения $(A(t))^{m}$, где $A(t)=1+a_{1} t+a_{2} t^{2}+\cdots, a_{i}$ и $m$ - элементы из кольца Гротендика $K_{0}\left(\mathscr{V}_{\mathbb{C}}\right)$.

Это мотивирует следующее определение [63].

ОПРедЕЛЕНиЕ. Степенной структурой над (полу)кольцом $R$ с единицей называется отображение $(1+t R[[t]]) \times R \rightarrow 1+t R[[t]]:(A(t), m) \mapsto(A(t))^{m}$, обладающее следующими свойствами:

1) $(A(t))^{0}=1$

2) $(A(t))^{1}=A(t)$,

3) $(A(t) B(t))^{m}=(A(t))^{m}(B(t))^{m}$,

4) $(A(t))^{m+n}=(A(t))^{m}(A(t))^{n}$,

5) $(A(t))^{m n}=\left((A(t))^{n}\right)^{m}$,

6) $(1+t)^{m}=1+m t+($ члены более высокой степени),

7) $\left(A\left(t^{k}\right)\right)^{m}=\left.(A(t))^{m}\right|_{t \mapsto t^{k}}$.

ЗАмечАниЕ. Для кольца свойство 1) вытекает из остальных. Его необходимо сохранить только для полуколец. 
ОПРедЕЛЕНИЕ. Степенная структура над (полу)кольцом $R$ конечно определена, если для любого целого $K>0$ существует $L>0$ такое, что для любого $m \in R L$-струя ряда $A(t)$ определяет $K$-струю ряда $(A(t))^{m}$.

Нетрудно показать, что для задания конечно определенной степенной структуры над кольцом $R$ достаточно определить ряд $\left(A_{0}(t)\right)^{m}$ для любого фиксированного ряда $A_{0}(t)$ вида $1+t+$ (члены более высокой степени) (и для каждого $m \in R$ ) так, что:

1) $\left(A_{0}(t)\right)^{m}=1+m t+($ члены более высокой степени);

2) $\left(A_{0}(t)\right)^{m+n}=\left(A_{0}(t)\right)^{m}\left(A_{0}(t)\right)^{n}$.

Действительно, любой ряд $A(t) \in 1+t \cdot R[[t]]$ однозначно представим в виде

$$
A(t)=\prod_{i=1}^{\infty}\left(A_{0}\left(t^{i}\right)\right)^{b_{i}}
$$

с $b_{i} \in R$. Тогда

$$
(A(t))^{m}=\prod_{i=1}^{\infty}\left(A_{0}\left(t^{i}\right)\right)^{b_{i} m}
$$

Поэтому каждой пре- $\lambda$-структуре на кольце $R$ однозначно соответствует конечно определенная степенная структура над ним. C другой стороны, одна и та же степенная структура соответствует множеству пре- $\lambda$-структур. В качестве ряда $A_{0}(t)$ удобно использовать ряд $(1-t)^{-1}=1+t+t^{2}+\cdots \in R[[t]]$.

Как было указано выше, некоторая (в каком-то смысле - естественная) пре- $\lambda$-структура на кольце Гротендика $K_{0}\left(\mathscr{V}_{\mathbb{C}}\right)$ определяется дзета-функцией Капранова $\zeta_{X}(t)$. Этой пре- $\lambda$-структуре соответствует степенная структура над кольцом $K_{0}\left(\mathscr{V}_{\mathbb{C}}\right)$, определенная формулой $(16)$. Оказывается, что эта степенная структура имеет красивое геометрическое описание. Это описание позволяет использовать указанную степенную структуру для формулировки и доказательства некоторых соотношений между производящими рядами классов (в кольце $\left.K_{0}\left(\mathscr{V}_{\mathbb{C}}\right)\right)$ конфигурационных пространств и их инвариантов.

Геометрическое описание степенной структуры, определяемой дзета-функцией Капранова, состоит в следующем. Пусть $A(t)=1+\left[A_{1}\right] t+\left[A_{2}\right] t^{2}+\cdots$, где $A_{i}$ - (комплексные) конструктивные множества, и $M$ - тоже конструктивное множество. Пусть $\mathfrak{A}$ - несвязное объединение $\bigsqcup_{i=1}^{\infty} A_{i}$, и пусть $I: \mathfrak{A} \rightarrow \mathbb{Z}-$ тавтологическая функция, принимающая значение $i$ на $A_{i} \subset \mathfrak{A}$. Тогда коэффициент при $t^{k}$ в ряде $(A(t))^{[M]}$ представлен конфигурационным пространством пар $(K, \varphi)$, где $K$ - конечное подмножество пространства $M$, а $\varphi$ - отображение из $K$ в $\mathfrak{A}$ такое, что $\sum_{x \in K} I(\varphi(x))=k$.

Чтобы описать такое конфигурационное пространство как конструктивное множество, можно записать его в виде

$$
\sum_{\underline{k}: \sum i k_{i}=k}\left[\left(\left(\prod_{i} M^{k_{i}}\right) \backslash \Delta\right) \times \prod_{i} A_{i}^{k_{i}} / \prod_{i} S_{k_{i}}\right],
$$


где $\underline{k}=\left\{k_{i}: i \in \mathbb{Z}_{>0}, k_{i} \in \mathbb{Z}_{\geqslant 0}\right\}$ - разбиение числа $k, \Delta$ - "большая диагональ" в $M^{\Sigma} k_{i}$, состоящая из наборов из $\left(\sum k_{i}\right)$ точек пространства $M$, из которых по крайней мере две совпадают; группа перестановок $S_{k_{i}}$ действует одновременной перестановкой соответствующих $k_{i}$ сомножителей в $\prod_{i} M^{k_{i}} \supset\left(\prod_{i} M^{k_{i}}\right) \backslash \Delta$ и пространств $A_{i}$. (Заметим, что факторпространство квазипроективного пространства по действию конечной группы тоже является квазипроективным. Соотношение между этой формулой и приведенным выше описанием понятно.)

Можно показать (см. [63]), что описанная операция действительно определяет степенную структуру над кольцом $K_{0}\left(\mathscr{V}_{\mathbb{C}}\right)$, т. е. она удовлетворяет условиям 1)-7) определения. Тот факт, что эта структура соответствует дзетафункции Капранова, вытекает из формулы

$$
\left(1+t+t^{2}+\cdots\right)^{[M]}=1+[M] t+\left[S^{2} M\right] t^{2}+\left[S^{3} M\right] t^{3}+\cdots .
$$

Действительно, поскольку существует только одно отображение из множества $K$ в точку (коэффициент в ряде $\left.1+t+t^{2}+\cdots\right)$, то коэффициент при $t^{k}$ в левой части равенства (18) представлен пространством, точка которого - это конечное множество точек из пространства $M$ с положительными кратностями такими, что сумма этих кратностей равна $k$. Это в точности определение $k$-й симметрической степени пространства $M$.

Также полезно описать бином $(1+t)^{[M]}$. Коэффициент при $t^{k}$ в нем представлен пространством, точка которого - это конечное подмножество пространства $M$, состоящее из $k$ элементов, т. е. конфигурационным пространством $\left(M^{k} \backslash \Delta\right) / S_{k}$ неупорядоченных наборов из $k$ различных точек пространства $M$.

Эйлерова характеристика и многочлен Ходжа-Делиня являются гомоморфизмами кольца Гротендика $K_{0}\left(\mathscr{V}_{\mathbb{C}}\right)$ в кольцо $\mathbb{Z}$ целых чисел и в кольцо $\mathbb{Z}[u, v]$ многочленов от двух переменных соответственно. Пусть $R_{1}$ и $R_{2}$ - кольца со степенными структурами над ними. Гомоморфизм колец $\varphi: R_{1} \rightarrow R_{2}$ индуцирует естественный гомоморфизм $R_{1}[[t]] \rightarrow R_{2}[[\underline{t}]]$ (также обозначаемый $\varphi$ ) по формуле

$$
\varphi\left(\sum a_{i} t^{i}\right)=\sum \varphi\left(a_{i}\right) t^{i}
$$

Говорят, что гомоморфизм $\varphi$ является гомоморфизмом степенных структур, если $(\varphi(A(t)))^{\varphi(m)}=\varphi\left((A(t))^{m}\right)$ для $A(t) \in 1+t R[[t]], m \in R$. Имеет место следующее простое утверждение.

ПредлОжениЕ 4. Если степенные структуръ над колъцами $R_{1}$ u $R_{2}$ конечно определенные, а гомоморфизм колеи, $\varphi: R_{1} \rightarrow R_{2}$ таков, что

$$
(1-t)^{-\varphi(m)}=\varphi\left((1-t)^{-m}\right)
$$

для любого $m \in R$, то ч является гомоморфизмом степенных структур.

Над кольцами $\mathbb{Z}$ целых чисел и $\mathbb{Z}\left[u_{1}, \ldots, u_{r}\right]$ многочленов от $r$ переменных имеются естественные степенные структуры, относительно которых эйлерова характеристика и многочлен Ходжа-Делиня являются гомоморфизмами степенных структур. Над кольцом $\mathbb{Z}\left[u_{1}, \ldots, u_{r}\right]$ степенная структура определяется 
следующим образом. Пусть

$$
P(\underline{u})=P\left(u_{1}, \ldots, u_{r}\right)=\sum_{\underline{k} \in \mathbb{Z}_{\geqslant 0}^{r}} p_{\underline{k}} \underline{u}^{\underline{k}} \in \mathbb{Z}\left[u_{1}, \ldots, u_{r}\right],
$$

где $\underline{u}=\left(u_{1}, \ldots, u_{r}\right), \underline{k}=\left(k_{1}, \ldots, k_{r}\right), \underline{u} \underline{k}=u_{1}^{k_{1}} \cdots u_{r}^{k_{r}}, p_{\underline{k}} \in \mathbb{Z}$. Тогда

$$
(1-t)^{-P(\underline{u})}:=\prod_{\underline{k} \in \mathbb{Z}_{\geqslant 0}^{r}}\left(1-\underline{u}^{\underline{k}} t\right)^{-p_{\underline{k}}},
$$

где степень в правой части формулы (с целым показателем - $p_{k}$ ) имеет обычный смысл.

То, что эйлерова характеристика - гомоморфизм степенных структур, является утверждением леммы 2. Следующее утверждение (см., например, [64], [65]) означает, что гомоморфизмом степенных структур является многочлен Ходжа-Делиня.

ПРЕДЛОЖЕНИЕ 5. Имеет место равенство

$$
e\left((1-T)^{-[X]}\right)=(1-T)^{-e_{X}(u, v)}
$$

где степени понимаются в соответствии со степенными структурами над соответствующими кольцами: $K_{0}\left(\mathscr{V}_{\mathbb{C}}\right)$ u $\mathbb{Z}[u, v]$.

\section{0. Степенная структура над кольцом $K_{0}\left(\mathscr{V}_{\mathbb{C}}\right)$ и производящие ряды классов некоторых конфигурационных пространств}

Выражения, сформулированные в терминах степенной структуры над кольцом Гротендика квазипроективных многообразий, оказываются полезными как для записи формул для производящих рядов классов (в кольце $\left.K_{0}\left(\mathscr{V}_{\mathbb{C}}\right)\right)$ некоторых конфигурационных пространств, так и для их доказательства. Простейшими формулами такого типа являются указанные выше выражения для производящего ряда классов симметрических степеней пространства $X$ :

$$
\zeta_{X}(t)=\sum_{n=0}^{\infty}\left[S^{n} X\right] t^{n}=(1-t)^{-[X]},
$$

и для производящего ряда классов конфигурационных пространств $X_{n}=$ $\left(X^{n} \backslash \Delta\right) / S_{n} n$-точечных подмножеств пространства $X$ :

$$
\sum_{n=0}^{\infty}\left[X_{n}\right] t^{n}=(1+t)^{[X]}
$$

Более сложные и содержательные примеры возникают при рассмотрении схем Гильберта нульмерных подсхем на неособых многообразиях.

Пусть $\operatorname{Hilb}_{X}^{n}, n \geqslant 1,-$ схема Гильберта нульмерных подсхем длины $n$ на комплексном квазипроективном пространстве $X$ (см., например, [66]); для $x \in X$ 
пусть $\operatorname{Hilb}_{X, x}^{n}$ - схема Гильберта подсхем на $X$, сконцентрированных в точке $x$. Пусть

$$
\mathbb{H}_{X}(T):=1+\sum_{n=1}^{\infty}\left[\operatorname{Hilb}_{X}^{n}\right] T^{n}, \quad \mathbb{H}_{X, x}(T):=1+\sum_{n=1}^{\infty}\left[\operatorname{Hilb}_{X, x}^{n}\right] T^{n}
$$

- производящие ряды классов схем Гильберта $\operatorname{Hilb}_{X}^{n}$ и $\mathrm{Hilb}_{X, x}^{n}$ в полукольце Гротендика $S_{0}\left(\mathscr{V}_{\mathbb{C}}\right)$ или в кольце $K_{0}\left(\mathscr{V}_{\mathbb{C}}\right)$, $\mathbb{A}^{d}-$ комплексное аффинное пространство размерности $d$.

Вычисление инвариантов схем Гильберта $\mathrm{Hilb}_{X}^{n}$ для неособого многообразия $X$ размерности $d$ может производиться в два шага. На первом шаге вычисляются соответствующие инварианты в локальном случае, т. е. инварианты схем Гильберта $\mathrm{Hilb}_{\mathbb{A}^{d}, 0}^{n}$ (или схем $\mathrm{Hilb}_{\mathbb{A}^{d}}^{n}$ ), а на втором локальные результаты собираются вместе для получения глобальных. Следующее утверждение формализует второй шаг (для инвариантов классов в (полу)кольце Гротендика квазипроективных многообразий).

Теорема 8 [67]. Для неособого квазипроективного многообразия $X$ размерности $d$ в полукольце $S_{0}\left(\mathscr{V}_{\mathbb{C}}\right)[[T]]$ ( и в кольце $\left.K_{0}\left(\mathscr{V}_{\mathbb{C}}\right)[[T]]\right)$ имеет место следующее равенство:

$$
\mathbb{H}_{X}(T)=\left(\mathbb{H}_{\mathbb{A}^{d}, 0}(T)\right)^{[X]} .
$$

ДокАзАтельство. Для локально замкнутого подпространства $Y \subset X$ обозначим через $\operatorname{Hilb}_{X, Y}^{n}$ схему Гильберта нульмерных подсхем длины $n$ на $X$, сконцентрированных в точках подпространства $Y$. Пусть

$$
\mathbb{H}_{X, Y}(T):=1+\sum_{n=1}^{\infty}\left[\operatorname{Hilb}_{X, Y}^{n}\right] T^{n}
$$

- соответствующий производящий ряд. Если $Y$ - замкнутое по Зарискому подмножество многообразия $X$, то $\mathbb{H}_{X}(T)=\mathbb{H}_{X, Y}(T) \cdot \mathbb{H}_{X, X \backslash Y}(T)$. (Это следует из того, что нульмерная подсхема на $X$ является объединением двух нульмерных подсхем, сконцентрированных на $Y$ и $X \backslash Y$ соответственно.) Поэтому достаточно доказать, что

$$
\mathbb{H}_{X, Y}(T)=\left(\mathbb{H}_{\mathbb{A}^{d}, 0}(T)\right)^{[Y]}
$$

для элементов $Y$ некоторого открытого (по Зарискому) покрытия многообразия $X$ и для их пересечений. Не теряя общности, можно предполагать, что каждый элемент такого покрытия лежит в аффинном пространстве $\mathbb{A}^{N}$ и первые $d$ аффинных координат $x_{1}, \ldots, x_{d}$ на $\mathbb{A}^{N}$ определяют локальные координаты на $X$ в каждой точке подпространства $Y$. (Это означает, что в каждой точке подпространства $Y$ проекция многообразия $X$ на соответствующее координатное подпространство невырождена.) Для точки $p=\left(x_{1}^{(0)}, \ldots, x_{d}^{(0)}, \ldots, x_{N}^{(0)}\right) \in Y$ нульмерная подсхема длины $n$, сконцентрированная в точке $p$, может быть определена уравнениями на $x_{1}-x_{1}^{(0)}, \ldots, x_{d}-x_{d}^{(0)}$, и таким образом схема Гильберта $\operatorname{Hilb}_{X,\{p\}}^{n}$ нульмерных подсхем длины $n$ на $X$, сконцентрированных в точке $p$, может быть отождествлена со схемой Гильберта Hilb $_{\mathbb{A}^{d}, 0}^{n}$. Поэтому 
нульмерная подсхема на многообразии $X$, сконцентрированная в точках подпространства $Y$, определяется конечным подмножеством $K \subset Y$, каждой точке $x$ которого соответствует нульмерная подсхема (стандартного) аффинного пространства $\mathbb{A}^{d}$, сконцентрированная в начале координат. При этом длина подсхемы равна сумме длин соответствующих подсхем на $\left(\mathbb{A}^{d}, 0\right)$. Теперь $(20)$ немедленно вытекает из геометрического описания степенной структуры над (полу)кольцом Гротендика квазипроективных многообразий. Теорема 8 доказана.

Поскольку $\left[\mathbb{A}^{d}\right]=\mathbb{L}^{d}$ и поэтому $\mathbb{H}_{\mathbb{A}^{d}}(T)=\left(\mathbb{H}_{\mathbb{A}^{d}, 0}(T)\right)^{\mathbb{L}^{d}}$, имеет место следующее утверждение.

СЛЕДСТВИЕ. Для неособого квазипроективного многообразия Х размерности d в кольце $K_{0}\left(\mathscr{V}_{\mathbb{C}}\right)\left[\mathbb{L}^{-1}\right][[T]]$ выполняется следующее равенство:

$$
\mathbb{H}_{X}(T)=\left(\mathbb{H}_{\mathbb{A}^{d}}(T)\right)^{\mathbb{L}^{-d}[X]} .
$$

Применяя соответствующие гомоморфизмы степенных структур, можно получить специализации формулы (19) для эйлеровой характеристики и для многочлена Ходжа-Делиня. В частности, имеет место следующее утверждение.

ПРЕДЛОЖЕНИЕ 6. Для неособого квазипроективного многообразия Х размерности d

$$
\chi\left(\mathbb{H}_{X}(T)\right)=\left(\chi\left(\mathbb{H}_{\mathbb{A}^{d}, 0}(T)\right)\right)^{\chi(X)} .
$$

Для многочлена Ходжа-Делиня имеем следующее утверждение.

Теорема 9. Для неособого квазипроективного многообразия Х размерноcmu d

$$
e\left(\mathbb{H}_{X}(T)\right)=\left(e\left(\mathbb{H}_{\mathbb{A}^{d}, 0}(T)\right)\right)^{e(X)},
$$

где в правой части формуль степенъ понимается в смысле степенной структуры над кольцом многочленов от двух переменных, описанной в конце раздела 9.

Это утверждение является основным результатом работы [65], записанным в более инвариантных терминах.

Для $d=\operatorname{dim} X=2$ теорема 8 с учетом результатов работы [68] дает

$$
\mathbb{H}_{X}(T)=\left(\prod_{n=1}^{\infty} \frac{1}{1-\mathbb{L}^{n-1} T^{n}}\right)^{[X]}
$$

Это - основной результат работы [69].

Если $X-d$-мерное квазипроективное пространство с изолированными особенностями в точках $x_{i}, i=1, \ldots, s$, то те же самые аргументы дают

$$
\mathbb{H}_{X}(T)=\left(\mathbb{H}_{\mathbb{A}^{d}, 0}(T)\right)^{\left[X \backslash\left\{x_{i}\right\}\right]} \prod_{i=1}^{s} \mathbb{H}_{X, x_{i}}(T) .
$$

Это равенство может быть записано в виде

$$
\mathbb{H}_{X}(T)=\int_{X} \mathbb{H}_{X, x}(T)^{d \chi_{g}}
$$


В общем случае (для квазипроективных пространств с неизолированными особенностями) равенство (22) не выполняется. Более того, $\mathbb{H}_{X, x}(T) \in K_{0}\left(\mathscr{V}_{\mathbb{C}}\right)[[T]]$ как функция от $x$ не обязана быть конструктивной (например, если особенности пространства $X$ имеют модули). Для выполнения равенства типа (22) нужно "отождествление особенностей" вдоль стратов некоторой стратификации пространства $X$.

А. Обломков и В. Шенде заметили, что при $t_{1}=\cdots=t_{r}=t$ ряд Пуанкаре $P_{\left\{v_{i}\right\}}(\underline{t})$ фильтрации, соответствующей особенности $C$ плоской кривой (а следовательно, и многочлен Александера от одной переменной $\Delta_{C}(t, \ldots, t)$ алгебраического зацепления, соответствующего кривой, раздел 6) равен производящему ряду эйлеровых характеристик частей схем Гильберта нульмерных подсхем на особенности кривой $C$, состоящих из подсхем, задаваемых главными идеалами. Они предложили гипотетическую формулу для производящего ряда эйлеровых характеристик самих схем Гильберта в терминах многочлена HOMFLY зацепления: [70].

\section{Список литературы}

[1] O. Y. Viro, "Some integral calculus based on Euler characteristic", Topology and geometry - Rohlin Seminar, Lecture Notes in Math., 1346, Springer, Berlin, 1988, $127-138$.

[2] M. Kontsevich, "Motivic integration", Lecture at Orsay, 1995.

[3] J. Denef, F. Loeser, "Motivic Igusa zeta functions", J. Algebraic Geom., 7:3 (1998), 505-537; arXiv: math/9803040.

[4] J. Denef, F. Loeser, "Germs of arcs on singular algebraic varieties and motivic integration", Invent. Math., 135:1 (1999), 201-232.

[5] J. Denef, F. Loeser, "Motivic integration, quotient singularities and the McKay correspondence", Compos. Math., 131:3 (2002), 267-290.

[6] V. V. Batyrev, D.I. Dais, "Strong McKay correspondance, string-theoretic Hodge numbers and mirror symmetry", Topology, 35:4 (1996), 901-929.

[7] V. V. Batyrev, L. A. Borisov, "Mirror duality and string-theoretic Hodge numbers", Invent. Math., 126:1 (1996), 183-203.

[8] V. V. Batyrev, "Non-Archimedean integrals and stringy Euler numbers of log-terminal pairs", J. Eur. Math. Soc. (JEMS), 1:1 (1999), 5-33.

[9] V. Batyrev, "Birational Calabi-Yau $n$-folds have equal Betti numbers", New trends in algebraic geometry (Warwick, 1996), London Math. Soc. Lecture Note Ser., 264, Cambridge Univ. Press, Cambridge, 1999, 1-11.

[10] M. Goresky, R. MacPherson, Stratified Morse theory, Ergeb. Math. Grenzgeb. (3), 14, Springer-Verlag, Berlin, 1988, ISBN: 3-540-17300-5, 272 pp.

[11] А. Н. Варченко, “Теоремы топологической эквисингулярности семейств алгебраических многообразий и семейств полиномиальных отображений”, Изв. АН СCCP. Сер. матем., 36:5 (1972), 957-1019; англ. пер.: А. N. Varčenko, "Theorems on the topological equisingularity of families of algebraic varieties and families of polynomial mappings", Math. USSR-Izv., 6:5 (1972), 949-1008.

[12] N. A'Campo, "La fonction zêta d'une monodromie", Comment. Math. Helv., 50:1 (1975), 233-248.

[13] Дж. Милнор, Особъе точки комплексных гиперповерхностей, Мир, М., 1971, 127 с.; пер. с англ.: J. Milnor, Singular points of complex hypersurfaces, Ann. of Math. Stud., 61, Princeton Univ. Press, Princeton, NJ; Univ. of Tokyo Press, Tokyo, 1968, 122 pp. 
[14] Lê D ung Tráng, "Some remarks on relative monodromy", Real and complex singularities, Proc. Ninth Nordic Summer School/NAVF Sympos. Math. (Oslo, 1976), Sijthoff and Noordhoff, Alphen aan den Rijn, 1977, 397-403.

[15] В.И. Арнольд, А.Н. Варченко, С.М.Гусейн-Заде, Особенности дифферениируемых отображений, т. II, Монодромия и асимптотики интегралов, Наука, M., 1984, 336 с.; англ. пер.: V.I. Arnol'd, S. M. Gusein-Zade, A. N. Varchenko, Singularities of differentiable maps, v. II, Monodromy and asymptotics of integrals, Monogr. Math., 83, Birkhäuser, Boston, MA, 1988, viii+492 pp.

[16] S. M. Gusein-Zade, I. Luengo, A. Melle-Hernández, "Partial resolutions and the zeta-function of a singularity", Comment. Math. Helv., 72:2 (1997), 244-256.

[17] S. A. Broughton, "On the topology of polynomial hypersurfaces", Singularities, Part 1 (Arcata, CA, 1981), Proc. Sympos. Pure Math., 40, Amer. Math. Soc., Providence, RI, 1983, 167-178.

[18] С. М. Гусейн-Заде, И. Луенго, А. Мелье-Эрнандез, “Дзета-функции ростков мероморфных функций и диаграмма Ньютона", Функи. анализ и его прил., 32:2 (1998), 26-35; англ. пер.: S. M. Gusein-Zade, I. Luengo, A. Melle-Hernández, "Zeta functions for germs of meromorphic functions, and Newton diagrams", Funct. Anal. Appl., 32:2 (1998), 93-99; arXiv: math/9705227.

[19] S. M. Gusein-Zade, I. Luengo, A. Melle-Hernández, "Bifurcations and topology of meromorphic germs", New developments in singularity theory (Cambridge, 2000), NATO Sci. Ser. II Math. Phys. Chem., 21, Kluwer Acad. Publ., Dordrecht, 2001, 279-304.

[20] С. М. Гусейн-Заде, И. Луенго, А. Мелье-Эрнандез, "О топологии ростков мероморфных функций и ее приложениях", Алгебра и анализ, 11:5 (1999), 92-99; англ. пер.: S. M. Gusein-Zade, I. Luengo, A. Melle-Hernández, "On the topology of germs of meromorphic functions and its applications", St. Petersburg Math. J., 11:5 (2000), $775-780$.

[21] S. M. Gusein-Zade, I. Luengo, A. Melle-Hernández, "On the zeta-function of a polynomial at infinity", Bull. Sci. Math., 124:3 (2000), 213-224.

[22] С. М. Гусейн-Заде, Д. Сирсма, “Деформации многочленов и их дзета-функции”, Труды Международной конференции по динамическим системам и дифференииальным уравнениям (5-10 июля 2004 г., Суздаль), Современная математика и ее приложения, 33, Академия наук Грузии, Институт кибернетики, Тбилиси, 2005, 36-42; англ. пер.: S. M. Gusein-Zade, D. Siersma, "Deformations of polynomials and their zeta-functions", J. Math. Sci. (N. Y.), 144:1 (2007), 3782-3788.

[23] С. М. Гусейн-Заде, Ф. Дельгадо, А. Кампильо, "Интегрирование по эйлеровой характеристике по пространству функций и полином Александера особенности плоской кривой", УМH, 55:6 (2000), 127-128; англ. пер.: S. M. Gusein-Zade, F. Delgado, A. Campillo, "Integration with respect to the Euler characteristic over a function space and the Alexander polynomial of a plane curve singularity", Russian Math. Surveys, 55:6 (2000), 1148-1149.

[24] A. Campillo, F. Delgado, S. M. Gusein-Zade, "The Alexander polynomial of a plane curve singularity and integrals with respect to the Euler characteristic", Internat. J. Math., 14:1 (2003), 47-54.

[25] A. Lemahieu, Poincaré series of embedded filtrations, arXiv: 0906.4184.

[26] A. Campillo, F. Delgado, K. Kiyek, "Gorenstein property and symmetry for one-dimensional local Cohen-Macaulay rings", Manuscripta Math., 83:1 (1994), 405-423.

[27] A. Campillo, F. Delgado, S. M. Gusein-Zade, F. Hernando, "Poincaré series of collections of plane valuations", Internat. J. Math. (в печати); arXiv: 0903.3835.

[28] A. Campillo, F. Delgado, S. M. Gusein-Zade, "The Alexander polynomial of a plane curve singularity via the ring of functions on it", Duke Math. J., 117:1 (2003), $125-156$. 
[29] I. G. Macdonald, "The Poincaré polynomial of a symmetric product", Math. Proc. Cambridge Philos. Soc., 58 (1962), 563-568.

[30] D. Eisenbud, W. Neumann, "Three-dimensional link theory and invariants of plane curve singularities", Ann. Math. Stud., 110, Princeton Univ. Press, Princeton, NJ, 1985, ISBN: 0-691-08380-0, 173 pp.

[31] С. М. Гусейн-Заде, Ф. Дельгадо, А. Кампильо, "О монодромии особенности плоской кривой и ряде Пуанкаре ее кольца функций", Функи. анализ и его прил., 33:1 (1999), 66-68; англ. пер.: S. M. Gusein-Zade, F. Delgado, A. Campillo, "On the monodromy of a plane curve singularity and the Poincare series of the ring of functions on the curve", Funct. Anal. Appl., 33:1 (1999), 56-57.

[32] С. М. Гусейн-Заде, Ф. Дельгадо, А. Кампильо, "Полином Александера особенности плоской кривой и кольцо функций на ней”, УМH, 54:3 (1999), 157-158; англ. пер.: S. M. Gusein-Zade, F. Delgado, A. Campillo, "The Alexander polynomial of plane curve singularities and rings of functions on curves", Russian Math. Surveys, $\mathbf{5 4 : 3}$ (1999), 634-635.

[33] С. М. Гусейн-Заде, Ф. Дельгадо, А. Кампилло, “О монодромии плоской кривой на бесконечности и о рядах Пуанкаре кольца ее координат", Труды международной конференции, посвященной 90-летию со дня рождения Л.С. Понтрягина, Геометрия и топология-7 (31 августа - 6 сентября 1998 г., Москва), Итоги науки и техники. Сер. Соврем. матем. и ее прил. Темат. обз., 68, ВИНИТИ, М., 1999, 49-54; англ. пер.: S. M. Gusein-Zade, F. Delgado, A. Campillo, "On the monodromy at infinity of a plane curve and the Poincaré series of its coordinate ring", J. Math. Sci. (New York), 105:2 (2001), 1839-1842.

[34] A. Campillo, F. Delgado, S. M. Gusein-Zade, "Poincaré series of curves on rational surface singularities", Comment. Math. Helv., 80:1 (2005), 95-102.

[35] W. Ebeling, "Poincaré series and monodromy of a two-dimensional quasihomogeneous hypersurface singularity", Manuscripta Math., 107:3 (2002), 271-282.

[36] W. Ebeling, S. M. Gusein-Zade, "Poincaré series and zeta function of the monodromy of a quasihomogeneous singularity", Math. Res. Lett., 9:4 (2002), 509-513.

[37] W. Ebeling, S. M. Gusein-Zade, "Monodromies and Poincaré series of quasihomogeneous complete intersections", Abh. Math. Sem. Univ. Hamburg, 74:1 (2004), 175-179.

[38] F. Delgado, S. M. Gusein-Zade, "Poincaré series for several plane divisorial valuations", Proc. Edinb. Math. Soc. (2), 46:2 (2003), 501-509.

[39] A. Campillo, F. Delgado, S. M. Gusein-Zade, "Poincaré series of a rational surface singularity", Invent. Math., 155:1 (2004), 41-53.

[40] A. Campillo, F. Delgado, S. M. Gusein-Zade, "On Poincaré series of filtrations on equivariant functions of two variables", Mosc. Math. J., 7:2 (2007), 243-255.

[41] С. М. Гусейн-Заде, Ф. Дельгадо, А. Кампильо, "Универсальные абелевы накрытия рациональных особенностей поверхностей и мультииндексные фильтрации", Функи. анализ и его прил., 42:2 (2008), 3-10; англ. пер.: S. M. Gusein-Zade, F. Delgado, A. Campillo, "Universal Abelian covers of rational surface singularities and multi-index filtrations", Funct. Anal. Appl., 42:2 (2008), 83-88.

[42] A. Némethi, "Poincaré series associated with surface singularities", Singularities I, Contemp. Math., 474, Amer. Math. Soc., Providence, RI, 2008, 271-297.

[43] A. Némethi, The Seiberg-Witten invariants of negative definite plumbed 3-manifolds, arXiv: 1003.1254.

[44] P. Deligne, "Théorie de Hodge. II", Inst. Hautes Études Sci. Publ. Math., 1971, № 40, $5-57$.

[45] P. Deligne, "Théorie de Hodge. III", Inst. Hautes Études Sci. Publ. Math., 1974, № 44, $5-77$. 
[46] В.И. Данилов, А.Г. Хованский, "Многогранники Ньютона и алгоритм вычисления чисел Ходжа-Делиня", Изв. АН СССР. Сер. матем., 50:5 (1986), 925-945; англ. пер.: V.I. Danilov, A. G. Khovanskii, "Newton polyhedra and an algorithm for computing Hodge-Deligne numbers", Math. USSR-Izv., 29:2 (1987), 279-298.

[47] F. Bittner, "The universal Euler characteristic for varieties of characteristic zero", Compos. Math., 140:4 (2004), 1011-1032.

[48] D. Abramovich, K. Karu, K. Matsuki, J. Włodarczyk, "Torification and factorization of birational maps", J. Amer. Math. Soc., 15:3 (2002), 531-572.

[49] J. Włodarczyk, "Toroidal varieties and the weak factorization theorem", Invent. Math., 154:2 (2003), 223-331.

[50] A. Campillo, F. Delgado, S. M. Gusein-Zade, "Multi-index filtrations and generalized Poincaré series", Monatsh. Math., 150:3 (2007), 193-209.

[51] P. Ozsváth, Z. Szabó, "Holomorphic discs and knot invariants", Adv. Math., 186:1 (2004), 58-116.

[52] J. Rasmussen, "Knot polynomials and knot homologies", Geometry and topology of manifolds, Fields Inst. Commun., 47, Amer. Math. Soc., Providence, RI, 2005, 261-280; arXiv: math/0504045.

[53] E. Gorsky, Combinatorial computation of the motivic Poincaré series, arXiv: 0807.0491.

[54] A. Craw, "An introduction to motivic integration", Strings and geometry, Clay Math. Proc., 3, Amer. Math. Soc., Providence, RI, 2004, 203-225.

[55] E. Looijenga, "Motivic measures", Astérisque, 2002, № 276, 267-297.

[56] M. Mustaţă, "Jet schemes of locally complete intersection canonical singularities", Invent. Math., 145:3 (2001), 397-424.

[57] M. Mustaţă, "Singularities of pairs via jet schemes", J. Amer. Math. Soc., 15:3 (2002), 599-615.

[58] С. М. Гусейн-Заде, И. Луенго, А. Мелье-Эрнандез, "Интегрирование по пространству непараметризованных дуг и мотивные аналоги дзета-функции монодромии", Геометрическая топология, дискретная геометрия и теория множеств, Сборник статей, Тр. МИАН, 252, Наука, М., 2006, 71-82; англ. пер.: S. M. Gusein-Zade, I. Luengo, A. Melle-Hernández, "Integration over spaces of nonparametrized arcs and motivic versions of the monodromy zeta function", Proc. Steklov Inst. Math., 252:1 (2006), 63-73.

[59] J. Denef, F. Loeser, "Lefschetz numbers of iterates of the monodromy and truncated arcs", Topology, 41:5 (2002), 1031-1040.

[60] M. Kapranov, The elliptic curve in the S-duality theory and Eisenstein series for Kac-Moody groups, arXiv: math/0001005.

[61] M. Larsen, V. A. Lunts, "Motivic measures and stable birational geometry", Mosc. Math. J., 3:1 (2003), 85-95.

[62] D. Knutson, $\lambda$-rings and the representation theory of the symmetric group, Lecture Notes in Math., 308, Springer-Verlag, Berlin-New York, 1973, 203 pp.

[63] S. M. Gusein-Zade, I. Luengo, A. Melle-Hernández, "A power structure over the Grothendieck ring of varieties", Math. Res. Lett., 11:1 (2004), 49-57; arXiv: math/0407204.

[64] J. Burillo, "The Poincaré-Hodge polynomial of a symmetric product of compact Kähler manifolds", Collect. Math., 41:1 (1990), 59-69.

[65] J. Cheah, "On the cohomology of Hilbert schemes of points", J. Algebraic Geom., 5:3 (1996), 479-511. 
[66] H. Nakajima, Lectures on Hilbert schemes of points on surfaces, Univ. Lecture Ser., 18, Amer. Math. Soc., Providence, RI, 1999, ISBN: 0-8218-1956-9, 132 pp.

[67] S. M. Gusein-Zade, I. Luengo, A. Melle-Hernández, "Power structure over the Grothendieck ring of varieties and generating series of Hilbert schemes of points", Michigan Math. J., 54:2 (2006), 353-359.

[68] G. Ellingsrud, S. A. Strømme, "On a cell decomposition of the Hilbert scheme of points in the plane", Invent. Math., 91:2 (1988), 365-370.

[69] L. Göttsche, "On the motive of the Hilbert scheme of points on a surface", Math. Res. Lett., 8:5-6 (2001), 613-627.

[70] A. Oblomkov, V. Shende, The Hilbert scheme of a plane curve singularity and the HOMFLY polynomial of its link, arXiv: 1003.1568.

С. М. Гусейн-Заде (S. M. Gusein-Zade)

Московский государственный университет

им. М. В. Ломоносова

E-mail: sabir@mccme.ru
Поступила в редакцию

20.01.2010 